Draft VERSION SEPTEMBER 20, 2018

Preprint typeset using $\mathrm{LAT}_{\mathrm{E}} \mathrm{X}$ style AASTeX6 v. 1.0

\title{
INTERSTELLAR GAS-PHASE ELEMENT DEPLETIONS IN THE SMALL MAGELLANIC CLOUD: A GUIDE TO CORRECTING FOR DUST IN QSO ABSORPTION LINE SYSTEMS ${ }^{\text {a }}$ b
}

\author{
EDWARD B. JENKINS \\ Princeton University Observatory, Princeton, NJ, 08544-1001 \\ George Wallerstein \\ University of Washington, Seattle, Dept. of Astronomy, Seattle, WA 98195-1580
}

\begin{abstract}
We present data on the gas phase abundances for 9 different elements in the interstellar medium of the Small Magellanic Cloud (SMC), based on the strengths of ultraviolet absorption features over relevant velocities in the spectra of 18 stars within the SMC. From this information and the total abundances defined by the element fractions in young stars in the SMC, we construct a general interpretation on how these elements condense into solid form onto dust grains. As a group, the elements $\mathrm{Si}, \mathrm{S}, \mathrm{Cr}$, $\mathrm{Fe}, \mathrm{Ni}$, and $\mathrm{Zn}$ exhibit depletion sequences similar to those in the local part of our Galaxy defined by Jenkins (2009). The elements Mg and Ti deplete less rapidly in the SMC than in the Milky Way, and Mn depletes more rapidly. We speculate that these differences might be explained by the different chemical affinities to different existing grain substrates. For instance, there is evidence that the mass fractions of polycyclic aromatic hydrocarbons (PAHs) in the SMC are significantly lower than those in the Milky Way. We propose that the depletion sequences that we observed for the SMC may provide a better model for interpreting the element abundances in low metallicity Damped Lyman Alpha (DLA) and sub-DLA absorption systems that are recorded in the spectra of distant quasars and gamma ray burst afterglows.
\end{abstract}

Keywords: dust, extinction - ISM: abundances - galaxies: ISM - galaxies: individual (SMC) - quasars: absorption lines - ultraviolet: ISM

\section{INTRODUCTION}

When combined with theories of stellar evolution and nucleosynthesis, measurements of the abundances of elements in different parts of any galactic system reveal much about how it formed and evolved, how and when it exchanged gas with the intergalactic medium (both infall and outflow), and how its stellar populations changed with time. Stars of different masses, initial compositions, and age produce their own distinct imprint of element production (Wheeler et al. 1989 ; Timmes et al. 1995 ; McWilliam 1997 ; Chiappini et al. 1999 ; Matteucci 2003 ; Kobayashi et al. 2006). For our own Galaxy, objects are close enough that we can examine in great detail how different element groups, such as $\alpha$, Fe-peak, neutron-capture or cosmic ray spallation products, change from one location (or star) to the next. From theories of nucleosynthesis, we know that even- $\mathrm{Z}$ elements of intermediate mass, such as $\mathrm{Mg}$, S, Si, Ca, and probably $\mathrm{Ti}$ arise primarily from fundamental reactions in massive stars and core-collapse SNe, while the odd-Z elements such as $\mathrm{P}, \mathrm{Na}, \mathrm{Al}$, and $\mathrm{K}$ depend on having a neutron excess and thus are driven by the initial metallicities of the stars (Suess \& Urey 1956 ; Burbidge et al. 1957 ; Cameron 1957). Type 1a supernovae are mostly responsible for the production of the Fe-group elements ( $\mathrm{V}, \mathrm{Cr}, \mathrm{Mn}, \mathrm{Fe}, \mathrm{Co}$, and $\mathrm{Ni}$ ). In the studies of individual stars, we are able to trace the mix of these groups as a function of stellar ages (conventionally traced by their relative abundances of iron $[\mathrm{Fe} / \mathrm{H}]$ ), or by their memberships in dynamically distinct populations (thin disk, thick disk, bulge, etc.).

\footnotetext{
a Based on observations with the NASA/ESA Hubble Space Telescope and additional data obtained from the Data Archive at the Space Telescope Science Institute, which is operated by the Associations of Universities for Research in Astronomy, Incorporated, under NASA contract NAS5-26555. These observations are associated with program nr. 13778

b (C)2017. The American Astronomical Society. All rights reserved.

ebj@astro.princeton.edu

walleg@u.washington.edu
} 
For other galaxies, we do not have access to the tremendous level of detail that we have from nearby. Nevertheless, the global values of some element abundances and their gradients across the surfaces of the galaxies can still be studied from the spectroscopy of emission lines from H II regions (Thuan et al. 1995 ; Izotov \& Thuan 1999; Chen et al. 2005 ; Christensen et al. 2005 ; Ellison et al. 2005 ; Schulte-Ladbeck et al. 2005 ; Péroux et al. 2011 ; 2012 ; 2014). This information is useful for gaining a better understanding of the changes caused by internal processes, mass loss, and mergers from one galaxy to the next. However, to go to even greater distances and explore the elemental makeup of galaxies during early times in the history of our universe, i.e., at redshifts $z \gtrsim 2.5$, it becomes difficult to obtain much spectroscopic detail from the light that is emitted. Virtually all of our knowledge on element abundances arises from studies of UV absorption lines, redshifted to visible wavelengths, that are seen in the spectra of background quasars or the afterglows of gamma ray bursts.

From UV absorption-line studies of interstellar material in our own Galaxy, we know that the gas-phase abundances of different elements are depleted by condensation into solid form within interstellar dust grains. ${ }^{1}$ We have known for some time that the strengths of these depletions vary strongly from one element to the next and from one sight line to another (Habing 1969 ; Wallerstein \& Goldsmith 1974; Morton 1975; Jenkins et al. 1986 ; Savage \& Sembach 1996 ; Dwek 2016). Researchers who study quasar absorption-line systems, such as Damped Lyman Alpha systems (DLAs having $\left.\log N(\mathrm{H} \mathrm{I}) / \mathrm{cm}^{-2} \gtrsim 20.3\right)$ or sub-DLAs $\left(18 \lesssim \log N(\mathrm{H} \mathrm{I}) / \mathrm{cm}^{-2} \lesssim 20.3\right)$ have had to use what we have learned from the depletion patterns in the Milky Way to make corrections that will help to define the pattern of the true, intrinsic abundances of any system (Pettini et al. 1994 ; Lu et al. 1996 ; Kulkarni et al. 1997 ; Pettini et al. 1997 ; Pettini et al. 1999 ; Prochaska \& Wolfe 1999 ; Pettini et al. 2000 ; Hou et al. 2001 ; Ledoux et al. 2002 ; Prochaska \& Wolfe 2002 ; Calura et al. 2003 ; Prochaska et al. 2003 ; Dessauges-Zavadsky et al. 2004 ; Vladilo 2004 ; Lopez et al. 2005 ; Dessauges- Zavadsky et al. 2006 ; Rodríguez et al. 2006 ; Levshakov et al. 2009 ; Meiring et al. 2009 ; Cooke et al. 2011 ; Rafelski et al. 2012 ; Som et al. 2013 ; Fox et al. 2014 ; Kulkarni et al. 2015 ; Prochaska et al. 2015 ; Som et al. 2015 ; Guber \& Richter 2016 ; Morrison et al. 2016 ; Quiret et al. 2016 ; Wiseman et al. 2016). Some early attempts to characterize the depletions of certain elements in order to correct for them were devised by Savaglio (2001), Vladilo (2002) and Prochaska \& Wolfe (2002).

In a comprehensive review of the gas-phase abundances of 17 different elements in the interstellar medium in our local region of the Milky Way, Jenkins (2009) presented an analysis that showed how strongly each element depletes into solid form (dust) as the overall levels of depletions for the other elements change from one sight line to the next. A remarkable finding from this investigation was that the logarithms of the depletion factors of different elements tracked each other in linear fashions, but at different rates. This unified picture of depletion greatly simplified our understanding of how elements disappear from the gas phase and bind into solid form. Going further, we were able to learn from these results that the proportions of different the atomic constituents of grains change as the overall severity of depletions changed (Jenkins $2009 ; 2013)$.

\section{MOTIVATION}

In principle, one might suppose that it should be a simple matter to use our knowledge of how depletions behave in our Galaxy to make corrections for such processes in other environments. Indeed, from the lack of any better choice many investigations invoked this method to determine the total element abundances in distant systems, most of which had metallicities of order $1 / 300$ to $1 / 3$ solar (Dessauges-Zavadsky et al. 2006 ; Dessauges-Zavadsky et al. 2007 ; Rafelski et al. 2014 ; Quiret et al. 2016). However, evidence has accumulated that shows that the depletion patterns probably change when the basic element abundances or production sequences are different. For example, in the Milky Way Si and Fe deplete at about the same rate, and the gas-phase logarithmic abundance ratio relative to the solar one $[\mathrm{Si} / \mathrm{Fe}]_{\text {gas }} \approx 0.8$. However, Wolfe et al. $(2005)$ showed that without corrections for depletion $[\mathrm{Si} / \mathrm{Fe}]_{\text {gas }}$ starts at about 0.3 for DLAs with $[\mathrm{Si} / \mathrm{H}]_{\text {gas }} \lesssim-1.0$, and increases only slightly as the metallicities approach that of our Galaxy. After we correct for depletion, we would say that the intrinsic (i.e., gas plus dust) $[\mathrm{Si} / \mathrm{Fe}] \approx-0.5$ for these systems, a trend that is contrary to $[\mathrm{Si} / \mathrm{Fe}] \approx+0.5$ for stars with $[\mathrm{Fe} / \mathrm{H}]<-1.5$ in the Milky Way (Timmes et al. 1995). Likewise, Ledoux et al. (2002) stated that "The correlation between $[\mathrm{Mn} / \mathrm{Fe}]$ and $[\mathrm{Zn} / \mathrm{Fe}]$... cannot be accounted for by any dust depletion sequence: it implies either variations of the intrinsic Mn abundance relative to Fe from -0.3 to +0.1 dex and/or a relation between depletion level and metallicity." They also stated that "The variations of $[\mathrm{Ti} / \mathrm{Fe}]$ vs. $[\mathrm{Zn} / \mathrm{Fe}]$ cannot be fitted by a single dust depletion sequence either." These abnormalities may be explained by chemical considerations in pre- existing solids: for instance, Lodders (2003) presented examples where

${ }^{1}$ Contrary to some early misconceptions, the elements $\mathrm{O}, \mathrm{S}$, and $\mathrm{Zn}$ are $\underline{\text { not }}$ undepleted. Only N seems to be mostly undepleted. 
the condensation of the elements $\mathrm{Ni}$ and Ge depend on the presence of a host element Fe to create an alloy. Likewise, the formation of refractory compounds that contain $\mathrm{Zn}$ and $\mathrm{Mn}$, such as $\mathrm{Zn}_{2} \mathrm{SiO}_{4}, \mathrm{ZnSiO}_{3}$, or $\mathrm{Mn}_{2} \mathrm{SiO}_{4}$, are aided by host minerals such as forsterite and enstatite. In some galaxy environments where the ratio of $\alpha$-group to Fe-peak elements differs from that of our own, or where the previous buildup of some element groups diverged from that of the Milky Way, certain elements may have had more or less than their respective host compounds, thus altering the depletion rates in a way that is difficult to predict.

From the preceding discussion, we can see a clear need to investigate the depletion sequences in a low-metallicity system, much as Jenkins (2009) had done with solar metallicity gas in the local part of our Galaxy. The Small Magellanic Cloud (SMC) is a nearby dwarf irregular galaxy that presents an excellent opportunity to perform such a definition. It has a considerably lower metallicity than that of the Milky Way, and its chemical evolution history might possibly present a better match to more distant galaxies with metallicities $[\mathrm{M} / \mathrm{H}] \sim-1$ that are typically represented by DLAs at redshifts $z \lesssim 3.5$ (Rafelski et al. 2012). Moreover, most of its stars indicate foreground extinction curves that differ from those of stars in the Milky Way (Hutchings 1982 ; Bromage \& Nandy 1983 ; Prevot et al. 1984 ; Gordon \& Clayton 1998 ; Gordon et al. 2003 ; Cartledge et al. 2005 ; Maíz Apellániz \& Rubio 2012 ; Hagen et al. 2016), which suggests deviations in the distributions of dust grain sizes and/or compositions (Draine \& Lee 1984 ; Boulanger et al. 1994 ; Weingartner \& Draine 2001 ; Zubko et al. 2004 ; Zonca et al. 2015). This difference could be relevant to studies of abundances in DLAs, since their extinction curves are similar to that of the SMC (Murphy \& Bernet 2016). As with our Galaxy, we can measure the abundances of elements in young stars, although with less accuracy. These stellar abundances can serve as a standard for the combined element abundances in both gas and dust.

There have been a number of studies of ISM abundances in the SMC that have already been carried out using data from spectrographs on the Hubble Space Telescope (HST) and the Far Ultraviolet Spectroscopic Explorer (FUSE) (Roth \& Blades 1997 ; Welty et al. 1997 ; Koenigsberger et al. 2001 ; Mallouris et al. 2001 ; Welty et al. 2001 ; Sofia et al. 2006). A dispute on Si abundances between Sofia et al. (2006) and Welty et al. (2001) for a single sight line, amounting to $0.19 \mathrm{dex}$, highlights the difficulties that have been encountered previously. Tchernyshyov et al. (2015) performed a more comprehensive survey that derived abundances of the elements $\mathrm{Si}, \mathrm{P}, \mathrm{Cr}$, and Fe for many stars in the SMC using spectra recorded by the Cosmic Origins Spectrograph (COS) on the HST. (We compare our results to theirs in Section 9.2.) Our approach differs from previous ones by obtaining medium resolution STIS echelle data with broad wavelength coverages that enabled us to cover many transitions of differing strengths for a significant collection of sight lines.

\section{SELECTION OF TARGET STARS}

Our investigation of element depletions made use of 18 sight lines toward stars in the SMC. Table 1 lists the stars used in this study. Fourteen of the target stars were observed in our Cycle 22 observing program (46 orbits, Program ID $=13778$, E. Jenkins, PI) on the Hubble Space Telescope (HST). In selecting which stars to observe, we made use of the SMC interstellar titanium abundances reported by Welty \& Crowther (2010) as a guide for sampling a wide selection of relative depletions. As a supplement to our observations, an additional four stars were observed for other programs, which produced suitable spectra that were publicly available in the Mikulski Archive for Space Telescopes (MAST) maintained by the Space Telescope Science Institute. Nearly all of the stars had measurements of atomic and molecular hydrogen column densities reported by Welty et al. (2012) for gas only within the SMC. For most stars, the combined column densities of hydrogen in both atomic and molecular form exceeded $10^{21} \mathrm{~cm}^{-2}$; only 3 stars had lower values (see Table 3 in Section 6). For such high column densities, corrections for unseen ionization stages should be negligible (Vladilo et al. 2001). Another important criterion in selecting stars was to insure that the projected rotational velocities $v \sin i>50 \mathrm{~km} \mathrm{~s}^{-1}$, so that stellar features would not create confusing continuum levels.

\section{OBSERVING STRATEGY AND DATA ANALYSIS}

While it is usually desirable to obtain spectra at the highest possible wavelength resolution for analyzing interstellar absorptions, especially if the features are strong, we decided that a broad coverage in wavelength would register many different transitions of different strengths, which outweighed the importance of fully resolving the velocity structures within the lines. For this reason, we constructed our observing program to record spectra of the SMC stars using the medium resolution $(\lambda / \Delta \lambda=30,000-45,800)$ echelle modes $(\mathrm{E} 140 \mathrm{M}$ and $\mathrm{E} 230 \mathrm{M})$ of the Space Telescope Imaging Spectrograph (STIS) on the HST. Typical signal-to-noise ratios per resolution element ranged from about 10 at $1800 \AA$, to 30 at $1300 \AA$, and 40 at $2300 \AA$. 
Table 1. SMC Target Stars ${ }^{\mathrm{a}}$

\begin{tabular}{|c|c|c|c|c|c|c|c|c|}
\hline Star & $\begin{array}{c}\mathrm{AzV}^{\mathrm{b}} \\
(2)\end{array}$ & $\begin{array}{c}\text { R.A. (J2000) } \\
\left(\begin{array}{c}\mathrm{h} \mathrm{m} \mathrm{s} \\
(3)\end{array}\right.\end{array}$ & $\begin{array}{c}\text { Dec. }(\mathrm{J} 2000) \\
\left({ }^{\circ}{ }^{\prime \prime \prime}\right) \\
(4)\end{array}$ & $\begin{array}{c}V \\
(\mathrm{mag}) \\
(5)\end{array}$ & $(B-V)$ & $\begin{array}{c}E(B-V) \\
\text { Tot/SMC } \\
\quad(7)\end{array}$ & $\begin{array}{c}\text { Spectral } \\
\text { Type } \\
(8)\end{array}$ & $\begin{array}{c}\text { HST Obs. } \\
\operatorname{Pgm}(\mathrm{s}) .{ }^{\mathrm{c}} \\
(9)\end{array}$ \\
\hline Sk $13 \ldots$ & 18 & $\begin{array}{lll}0 & 47 & 12.2\end{array}$ & $-73 \quad 633$ & 12.44 & 0.03 & $0.20 / 0.16$ & B2 Ia & $1,2,3$ \\
\hline Sk $18 \ldots$ & 26 & 04750.0 & $\begin{array}{lll}-73 & 8 & 21\end{array}$ & 12.46 & -0.17 & $0.15 / 0.11$ & O7 III & 1,4 \\
\hline$\cdots$ & 47 & 04851.5 & -732559 & 13.44 & -0.18 & $0.13 / 0.09$ & O8 III & 1,5 \\
\hline HD $5045(\mathrm{Sk} 40)$ & 78 & 05038.4 & -732818 & 11.05 & -0.05 & $0.14 / 0.10$ & $\mathrm{~B} 1 \mathrm{Ia}+$ & 1 \\
\hline$\ldots$ & 80 & 05043.8 & -724742 & 13.32 & -0.13 & $0.19 / 0.15$ & O4-6n(f)p & 1,5 \\
\hline$\cdots$ & 95 & 05121.6 & -724415 & 13.78 & -0.18 & $0.14 / 0.10$ & O7 III & 1,5 \\
\hline$\ldots$ & 104 & 05138.4 & $-7248 \quad 6$ & 13.17 & -0.16 & $0.06 / 0.02$ & B0.5 Ia & 3 \\
\hline$\ldots$ & 207 & 05833.2 & -715547 & 14.25 & -0.20 & $0.12 / 0.09$ & $\mathrm{O} 7.5 \mathrm{~V}((\mathrm{f}))$ & 1 \\
\hline$\cdots$ & 216 & 05859.1 & -724434 & 14.22 & -0.13 & $0.13 / 0.09$ & B1 III & 3 \\
\hline HD 5980 (Sk 78) & 229 & 05926.6 & $-72 \quad 954$ & 11.85 & -0.23 & $0.07 / 0.04$ & WN6h & $6,7,8,9^{\mathrm{d}}$ \\
\hline Sk $85 \ldots$. & 242 & $\begin{array}{lll}1 & 0 & 6.9\end{array}$ & -721357 & 12.07 & -0.12 & $0.07 / 0.04$ & B1 Ia & 1 \\
\hline$\cdots$ & 321 & $1 \quad 257.1$ & $\begin{array}{lll}-72 & 8 & 9\end{array}$ & 13.76 & -0.19 & $0.12 / 0.09$ & O9 IInp & 1 \\
\hline Sk $108 \ldots$ & 332 & 1325.2 & $-72 \quad 644$ & 12.40 & -0.24 & $\cdots$ & WN3+O6.5(n) & $10,11^{\mathrm{e}}$ \\
\hline$\cdots$ & 388 & 1539.5 & -722927 & 14.09 & -0.21 & $0.11 / 0.08$ & $\mathrm{O} 4 \mathrm{~V}$ & 1 \\
\hline Sk $143 \ldots$ & 456 & 11055.8 & -724256 & 12.83 & 0.10 & $0.36 / 0.33$ & O9.7 Ib & 1,2 \\
\hline$\cdots$ & 476 & 11342.5 & -731730 & 13.52 & -0.09 & $0.23 / 0.20$ & $\mathrm{O} 6.5 \mathrm{~V}$ & 1 \\
\hline Sk 190 & $\cdots$ & 13128.0 & -732214 & 13.54 & -0.18 & $0.11 / 0.07$ & O8 Iaf & 1 \\
\hline Sk $191 \ldots \ldots \ldots$ & $\cdots$ & 14142.1 & -735038 & 11.85 & -0.04 & $0.14 / 0.10$ & B1.5 Ia & 1,3 \\
\hline
\end{tabular}

${ }^{a}$ Data and their sources are taken from Table 1 of Welty et al. (2012).

${ }^{b}$ Number in the catalog of Azzopardi \& Vigneau (1982).

${ }^{c}$ Observing program numbers and principal investigators: (1) 13778 (E. Jenkins), (2) 9383 (K. Gordon), (3) 9116 (D. Lennon), (4) 12978 (D. Welty), (5) 7437 (D. Lennon), (6) 7480 (G. Koenigsberger), (7) 9094 (G. Koenigsberger), (8) 11623 (G. Koenigsberger), (9) 13373 (G. Koenigsberger), (10) 4048 (J. Hutchings), (11) 5608 (D. Welty).

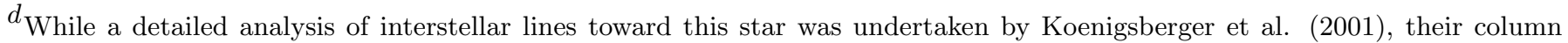
densities for the SMC features were derived using the formula for optically thin absorption, which we feel is inappropriate. Therefore, we derived all of the column densities independently.

${ }^{e}$ Column densities were not derived here, but instead were taken from Welty et al. (1997), Mallouris (2003) and Sofia et al. (2006). When appropriate, we adjusted column densities to reflect changes in some $f$-values.

With the exception of one star, AzV 95, the SMC absorption features were well separated from those of the disk and halo of our Galaxy. Our measurements of the lines included heliocentric velocities longward of $+60 \mathrm{~km} \mathrm{~s}^{-1}$, which is consistent with the lower velocity limit for the Ti II lines measured by Welty \& Crowther (2010). The upper velocity limits for most of the absorptions were at about $+190 \mathrm{~km} \mathrm{~s}^{-1}$, but the first 8 stars listed in Table 1 showed additional separate, narrow absorption at higher velocities, most of which reached up to about $+240 \mathrm{~km} \mathrm{~s}^{-1}$. In all cases, we determined column densities over the entire range of SMC velocities, so that they could be compared to the total amounts of hydrogen atoms and molecules. 
SMC ISM ELEMENT Depletions

Table 2. Transitions and SMC Stellar Abundances

\begin{tabular}{|c|c|c|c|c|c|c|}
\hline \multirow{2}{*}{$\begin{array}{c}\text { Element } \\
\qquad X\end{array}$} & \multirow{2}{*}{$\begin{array}{c}\lambda \\
(\AA)\end{array}$} & \multicolumn{2}{|c|}{$\log f \lambda^{\mathrm{a}}$} & \multicolumn{3}{|c|}{ SMC Total Abundances } \\
\hline & & Value & uncertainty & $\begin{array}{c}\text { Adopted Reference } \\
\log (X / \mathrm{H}) \odot^{\mathrm{b}}+11.35\end{array}$ & $\begin{array}{l}\text { Measured Deviations } \\
\text { from }[X / \mathrm{H}]^{\mathrm{b}}-0.65\end{array}$ & Sources $^{\mathrm{c}}$ \\
\hline (1) & $(2)$ & (3) & (4) & $(5)$ & $(6)$ & (7) \\
\hline $\mathrm{Mg}$ II & 1240 & -0.355 & $0.05^{\mathrm{d}}$ & 6.95 & $-0.21^{\mathrm{e}}$ & $1,2,3,4,5,6$ \\
\hline Si II & 1808 & 0.575 & 0.04 & 6.86 & -0.09 & $1,2,3,4,5,6$ \\
\hline \multirow[t]{2}{*}{ S II } & 1251 & $0.809^{\mathrm{f}}$ & 0.04 & 6.47 & 0.03 & $2,7,8,9$ \\
\hline & 1254 & $1.113^{\mathrm{f}}$ & 0.04 & & & \\
\hline $\mathrm{Ti} \mathrm{II}^{\mathrm{g}}$ & $\cdots$ & $\cdots$ & $\cdots$ & 4.30 & 0.08 & $8,10,11$ \\
\hline \multirow[t]{2}{*}{ Cr II } & 2056 & 2.326 & 0.024 & 4.99 & 0.12 & $7,8,10,11$ \\
\hline & 2066 & 2.024 & 0.025 & & & \\
\hline \multirow[t]{3}{*}{ Mn II } & 2577 & $2.966^{\mathrm{h}}$ & 0.006 & 4.78 & $0.23^{\mathrm{e}}$ & 8,11 \\
\hline & 2594 & $2.859^{\mathrm{h}}$ & 0.02 & & & \\
\hline & 2606 & $2.707^{\mathrm{h}}$ & 0.021 & & & \\
\hline \multirow[t]{5}{*}{ Fe II } & 1608 & 1.968 & $0.02^{\mathrm{d}}$ & 6.85 & -0.03 & $2,4,10,12$ \\
\hline & 2374 & 1.871 & 0.02 & & & \\
\hline & 2261 & 0.742 & 0.03 & & & \\
\hline & 2250 & 0.612 & 0.03 & & & \\
\hline & 1611 & 0.347 & 0.08 & & & \\
\hline \multirow[t]{6}{*}{ Ni II } & 1370 & $1.906^{\mathrm{i}}$ & 0.04 & 5.57 & 0.12 & $7,8,11$ \\
\hline & 1317 & $1.876^{\mathrm{i}}$ & 0.04 & & & \\
\hline & 1741 & 1.871 & 0.04 & & & \\
\hline & 1709 & 1.743 & 0.04 & & & \\
\hline & 1752 & 1.686 & 0.04 & & & \\
\hline & 1455 & 1.672 & 0.12 & & & \\
\hline Zn II & 2063 & $2.804^{\mathrm{j}}$ & $0.04^{\mathrm{d}}$ & 3.91 & 0.08 & 7,8 \\
\hline
\end{tabular}

${ }^{a}$ Unless otherwise noted, all values are taken from Morton (2003).

${ }^{b}$ Solar photospheric abundances adopted from values listed by Asplund et al. (2009). The value 11.35 arises from adding our adopted SMC logarithmic metallicity -0.65 to the standard representations based on $\mathrm{H}=12$.

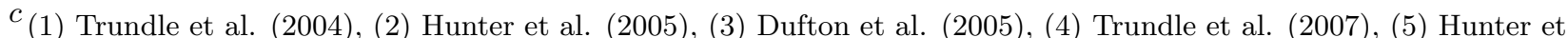
al. (2007), (6) Hunter et al. (2009), (7) Luck \& Lambert (1992), (8) Luck et al. (1998), (9) Rolleston et al. (2003), (10) Venn (1999), (11) Russell \& Dopita (1992), (12) Korn et al. (2000).

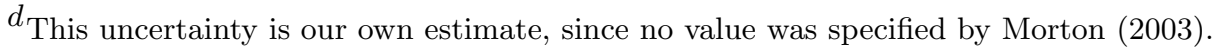

$e_{\text {See the text in Section } 5 .}$

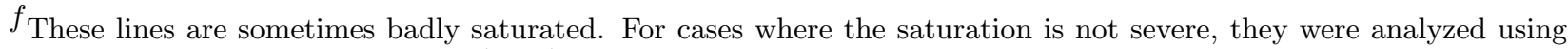
the technique devised by Jenkins (1996) to correct for unresolved saturated components. Revised $f$-values are from Kisielius et al. (2014).

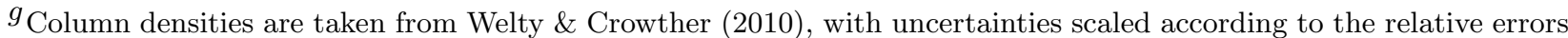
in the equivalent width measurements.

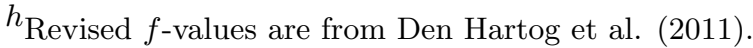

${ }^{i}$ Revised $f$-values are from Jenkins \& Tripp (2006).

$j$ Revised $f$-values are from Kisielius et al. (2015). We corrected for interference from an almost coincident Cr II line (2062.234 $\AA$, displaced from the Zn II line by $\Delta v=-62 \mathrm{~km} \mathrm{~s}^{-1}$ ) using the apparent optical depth information from another Cr II line at $2056 \AA$. 
Table 2 lists the transitions that were suitable for deriving column densities of various elements in their preferred stages of ionization for $\mathrm{H}$ I regions. The oscillator strengths ( $f$-values) for most transitions were taken from the compilation of Morton (2003), but with a few exceptions noted in the footnotes of the table. We derived column densities from the apparent optical depths (AOD) (Savage \& Sembach 1991) after the local parts of the spectra were renormalized to continuum levels defined by best-fitting Legendre polynomials (Sembach \& Savage 1992). In almost all cases, our analysis for transitions of different strengths for a single element yielded outcomes that agreed with each other to within the measurement uncertainties. Nevertheless, we acknowledge that our ability to uncover evidence of hidden saturated absorptions was somewhat hampered by our not being able to simultaneously measure transitions whose strengths differed by more than a factor of two.

Figure 1 shows the available absorption features for one of the stars in our survey, AzV 95, which is a typical case
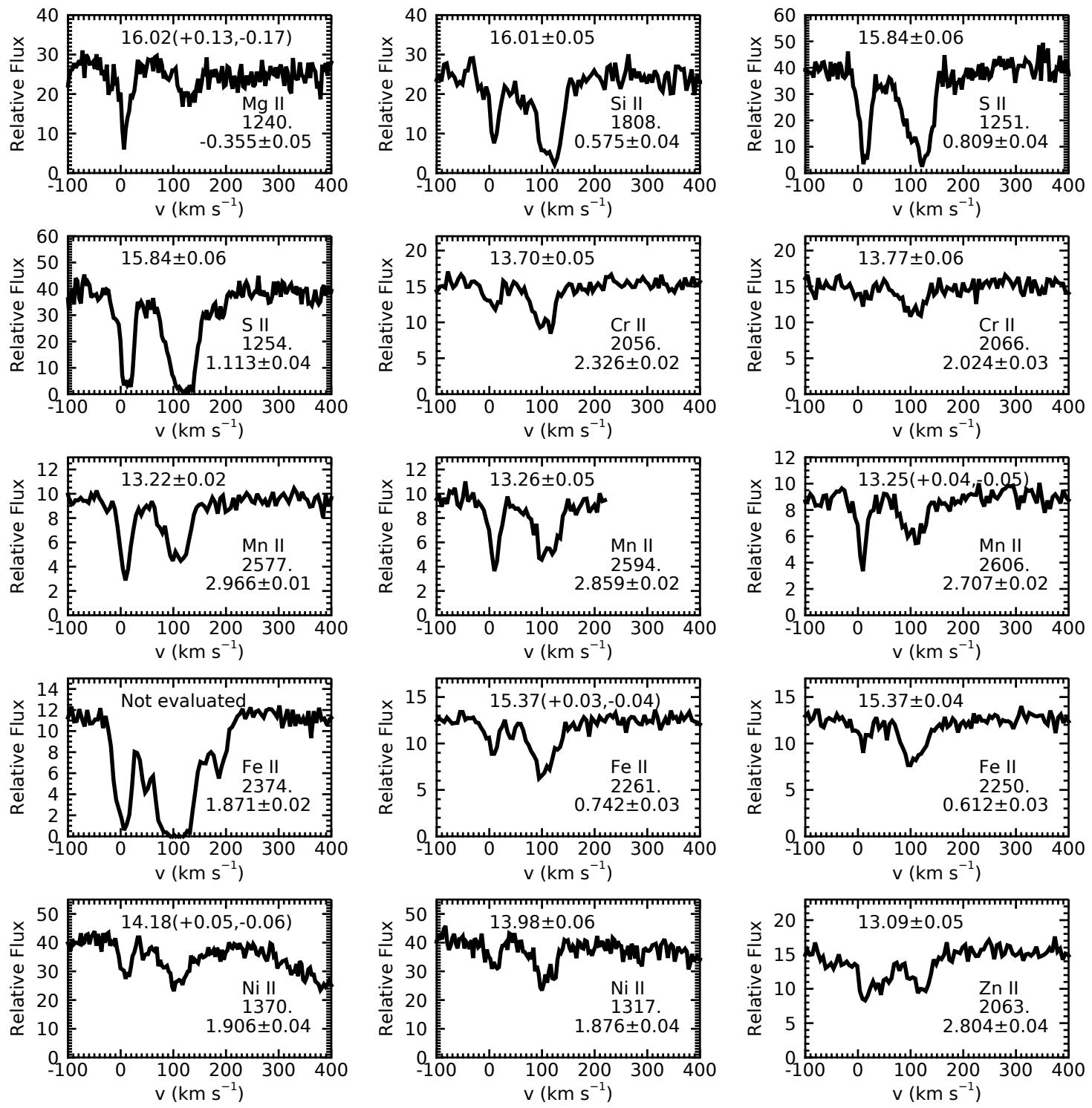

Figure 1. Absorption features for various elements in the spectrum of AzV 95, with the recorded intensities plotted as a function of heliocentric velocities. Near the top of each panel, we show our outcome for $\log (N)$ and its uncertainty limits. In the lower portion of each panel, we list the ion, together with the wavelength and value for $\log f \lambda$ for the transition. For this star, the outcomes for $\mathrm{Ni}$ represent the only case where the results for a single element differed by more than the uncertainties that we computed. The outcomes for the two transitions of S II are identical because both lines were analyzed together using the correction method of Jenkins (1996) for interpreting apparent optical depths that have unresolved saturated structures. We show the absorption by the very strong transition of Fe II at $2374 \AA$ simply to illustrate the range of velocities of even the smallest amounts of SMC gas for this star, some of which exhibits an overlap in velocity with material in our Galaxy. (This situation occurred only for this star however.) The absorption at $2374 \AA$ was much too saturated to derive a column density. 
from the standpoint of the star's brightness, hydrogen column density and our spectral coverage. Many transitions of other elements within our wavelength coverage could not be used. For instance, the strong transitions of C II, N I, O I and Si II were always completely saturated. Even the weak line of Si II at $1808 \AA$ was too saturated for deriving column densities for some stars. The two weaker lines (1251 and $1254 \AA$ ) of the S II triplet were sometimes only moderately saturated, which made them good candidates for analyzing their apparent optical depths after correcting for unresolved saturated structures using the method proposed by Jenkins (1996). At the other extreme, the intersystem lines of C II (2325 $\AA)$, Si II $(2335 \AA)$, and usually O I $(1356 \AA)$ were too weak to observe. Likewise, with very few exceptions we found that the allowed transitions of B II, Cu II, Ge II Ga II, and Kr I were too weak to measure above the noise. A feature of P II at $1152.82 \AA$ had a respectable strength, but for most stars our spectra at that wavelength had a very poor signal-to-noise ratio. Thus the outcomes would be uncertain and could also be subject to systematic errors (Fox et al. 2005).

For some transitions, we had to acknowledge and compensate for overlaps in absorption: the Mg II line at $1239.925 \AA$ covering the velocity range of the SMC gas suffered interference from the Milky Way absorption arising the other member of the doublet at $1240.395 \AA$. Thus, for deriving the column density of Mg II in the SMC, we had to rely entirely on the line at the longer wavelength. The transition of Zn II at $2026.137 \AA$ suffers interference from nearby lines of Cr II (2026.269 $)$ ), Co II (2026.412 $\AA$ ) and Mg I (2026.477 $)$. The Cr II and Co II lines are probably too weak to matter, but the Mg I line may make a non-negligible contribution. Thus, we chose not to use the $2026 \AA$ line of Zn II, chiefly because we could not correct for the Mg I absorption (another transition of Mg I at $2852.963 \AA$ that could have been used as a reference was outside our wavelength coverage). The other available line of Zn II at $2062.660 \AA$ also has interference from a Cr II transition (2062.234 $\AA$ ). Under most circumstances, the wavelength separation of the two lines is large enough to not cause a problem. However the range of velocities over which SMC absorptions occur result in some overlap. Hence, we had to subtract off the optical depths of the Cr II feature from those of Zn II, using as a reference rescaled versions of the optical depths that we obtained from the Cr II absorption at 2056.257 $\AA$.

For the column density outcome arising from any single transition, we assigned an error $\sigma$ that included uncertainties caused by (1) the noise in the absorption profile, (2) errors in defining the continuum level, and (3) the uncertainty of the $f$-value (see Column 4 of Table 2), all of which were combined in quadrature. Continuum uncertainties can have a large impact on the measurement accuracies of weak lines. We evaluated them from the expected formal uncertainties in the polynomial coefficients of the fit, as described by Sembach \& Savage (1992), and then we multiplied them by 2 in order to make an approximate allowance for additional uncertainties arising from our freedom in selecting the most appropriate polynomial order. We determined the effects of such uncertainties by evaluating the column densities at the lower and upper extremes for the continuum. When results from more than one transition of a given element were available, we evaluated a column density based on a weighted average, with the weights proportional to the respective inverse square errors $1 / \sigma_{i}^{2}$. The uncertainty in any such combined result was assigned a value equal to $\left(\sum_{i} \sigma_{i}^{-2}\right)^{-0.5}$. For the measurements of S II, we added in quadrature an additional error of 0.1 dex to account for possible inaccuracies in correcting for saturation. ${ }^{2}$

\section{STELLAR REFERENCE ABUNDANCES}

As we had discussed in Section 2, a key component of our investigation is the establishment of a set of reference element abundances against which we can compare our interstellar gas-phase abundances. The findings for stars that have recently formed out of the ISM of the SMC should serve as our best examples. We have avoided using abundances derived from emission lines in H II regions because dust grains could survive within them and hence their gas-phase abundances could be reduced.

We have examined about 30 papers that presented results for stellar abundances in the SMC. ${ }^{3}$ In narrowing the selection and making choices for defining our adopted abundances (which varied from one element to the next), we have favored results that came from relatively recent investigations that have had access to modern, large-aperture telescopes with more powerful spectrographs, improved stellar atmosphere and line-formation codes (we preferred NLTE calculations over LTE ones), and more accurate transition strengths. We have also recognized that the spectra of some types of stars are easier to interpret than others. For instance, main-sequence and giant B-type stars are relatively straightforward to analyze, while Cepheids have the complications of differential velocities in the pulsations that make the lines hard to interpret, and cool stars with surface convection require more elaborate modeling.

Some elements appear to have a small dispersion in the outcomes from different investigations $(\sigma \sim 0.15)$, such as

${ }^{2}$ Except for AzV 332, where we imported the value for $N$ (S II) from Mallouris (2003).

${ }^{3}$ In the appendix of the paper by Welty et al. (1997), there is a good summary of prior abundance determinations in the SMC. 
$\mathrm{Mg}, \mathrm{Si}, \mathrm{Ti}$, and $\mathrm{Cr}$, while the elements $\mathrm{Mn}, \mathrm{Fe}, \mathrm{Ni}$ and $\mathrm{Zn}$ either have few determinations or larger dispersions in the outcomes. Low dispersions of results do not necessarily indicate that the average results are reliable, since systematic errors could persist throughout many or all of the investigations (Blanco-Cuaresma et al. 2016 ; Hinkel et al. 2016). For instance, most of the stellar abundances in the SMC must be derived from spectra of luminous stars, for which the uncertainties of the model stellar atmospheres dominate the total uncertainties of the abundance determinations.

The elements in Table 2 can be divided into the $\alpha$ group; Mg, Si, S, and Ti and the Fe-peak species from $\mathrm{Cr}$ to $\mathrm{Zn}$. The dominant isotopes of the $\alpha$ elements have nuclei with an integral number of $\alpha$ particles, except for Ti, which also has a clump of 4 neutrons. In old, metal-poor stars, such as those in globular clusters and the Galactic thick disc and halo, there is a gradual increase of $\alpha$ elements relative to $\mathrm{Fe}$ as $[\mathrm{Fe} / \mathrm{H}]$ diminishes from 0.0 to about -1.0 . However, Mucciarelli (2014) found that this trend is displaced toward lower metallicities in the SMC, and that for $[\mathrm{Fe} / \mathrm{H}] \approx-0.7$, $[\alpha / \mathrm{Fe}] \approx 0$. If we take a straight mean of the $4 \alpha$ elements in Table 2 we find that $[\alpha / \mathrm{H}]=-0.7{ }^{4}$ This average does not include $\mathrm{C}$, which appears to have a much lower abundance (this is an issue that we will discuss in Section 9.4). For the 5 Fe-peak elements a straight mean of $[\mathrm{Fe} / \mathrm{H}]=-0.60$. Of the 5 Fe-peak elements the abundances of $\mathrm{Mn}$ and $\mathrm{Zn}$ are the least certain. Mn has relatively few determinations. The abundance of $\mathrm{Zn}$, which is featured in many studies of DLA gas compositions, depends on 2 lines of Zn I in Cepheids, the atmospheres of which are difficult to model because of the running wave that passes through them during each cycle. Even so, omitting Mn and $\mathrm{Zn}$ from the mean for the Fe group leaves it virtually unchanged at -0.58 .

In short, we feel that it is difficult to obtain elemental abundances in stars with an indisputable accuracy better than 0.1 dex. For this reason, we can simplify our reference standards by adopting for all elements a mean value of -0.65 for $[X / \mathrm{H}]$ that we obtained for the averages of the $\alpha$ and Fe-peak groups. ${ }^{5}$ These reference values are listed in Column 5 of Table 2. Deviations of the stellar measurements from our adopted reference abundances are listed in Column 6 of the table, followed by a column that indicates the sources of the averages in each case. The largest deviations are found for $\mathrm{Mg}$ and $\mathrm{Mn}$. A deficiency for $[\mathrm{Mg} / \mathrm{Fe}]$ is seen for nearby dwarf spheroidal galaxies for $[\mathrm{Fe} / \mathrm{H}] \gtrsim-1.0$ (Tolstoy et al. 2009), but this element is usually analyzed using only a blend of Mg II lines at $4481 \AA$ (e.g., Trundle et al. 2007), so its accuracy may not be as good as that of the other elements. The few sources of information on Mn yielded an average $[\mathrm{Mn} / \mathrm{H}]=-0.42$, which seems too high relative to other Fe-group elements. There are only a few lines of Mn II, and there are uncertainties for Mn I due to departures from LTE in their excitation and ionization. Also $\mathrm{Mn}$ is an odd element and the iron group is dominated by even elements. One expects to have $[\mathrm{Mn} / \mathrm{Fe}]>0$ only for extremely metal-poor stars.

While we recognized 4 different evaluations of the sulfur abundance, and the dispersion between them is moderate, we nevertheless find that the average result yields an outcome that is significantly lower than some of our interstellar abundances. We return to this issue later in this paper (Section 7.5).

Overall, to within the expected accuracy of the SMC stellar abundance determinations, we find that the Fe-peak elements appear to have about the same deficiencies below the solar abundance as the $\alpha$ elements. Thus, we can regard the SMC gas as having a dilute concentration of heavy elements, but with a relative pattern from one element to the next that does not seem to differ appreciably from the solar one.

\footnotetext{
${ }^{4}$ While $\mathrm{O}$ is not listed in Table 2, an average value for $[\mathrm{O} / \mathrm{H}]=-0.65$ is nearly consistent with the abundance deficiencies of other $\alpha$ elements.

${ }^{5}$ The source of $\mathrm{Zn}$ in the Galaxy is uncertain because it may be produced in either SN Ia, SN II, or by neutron capture in massive stars (Woosley et al. 2002 ; Sukhbold et al. 2016). Elements heavier than Zn are formed by neutron capture so their abundances may differ from those of the iron-group and the alpha elements. Unfortunately, their abundances are too low for us to measure in the spectra of our SMC stars.
} 


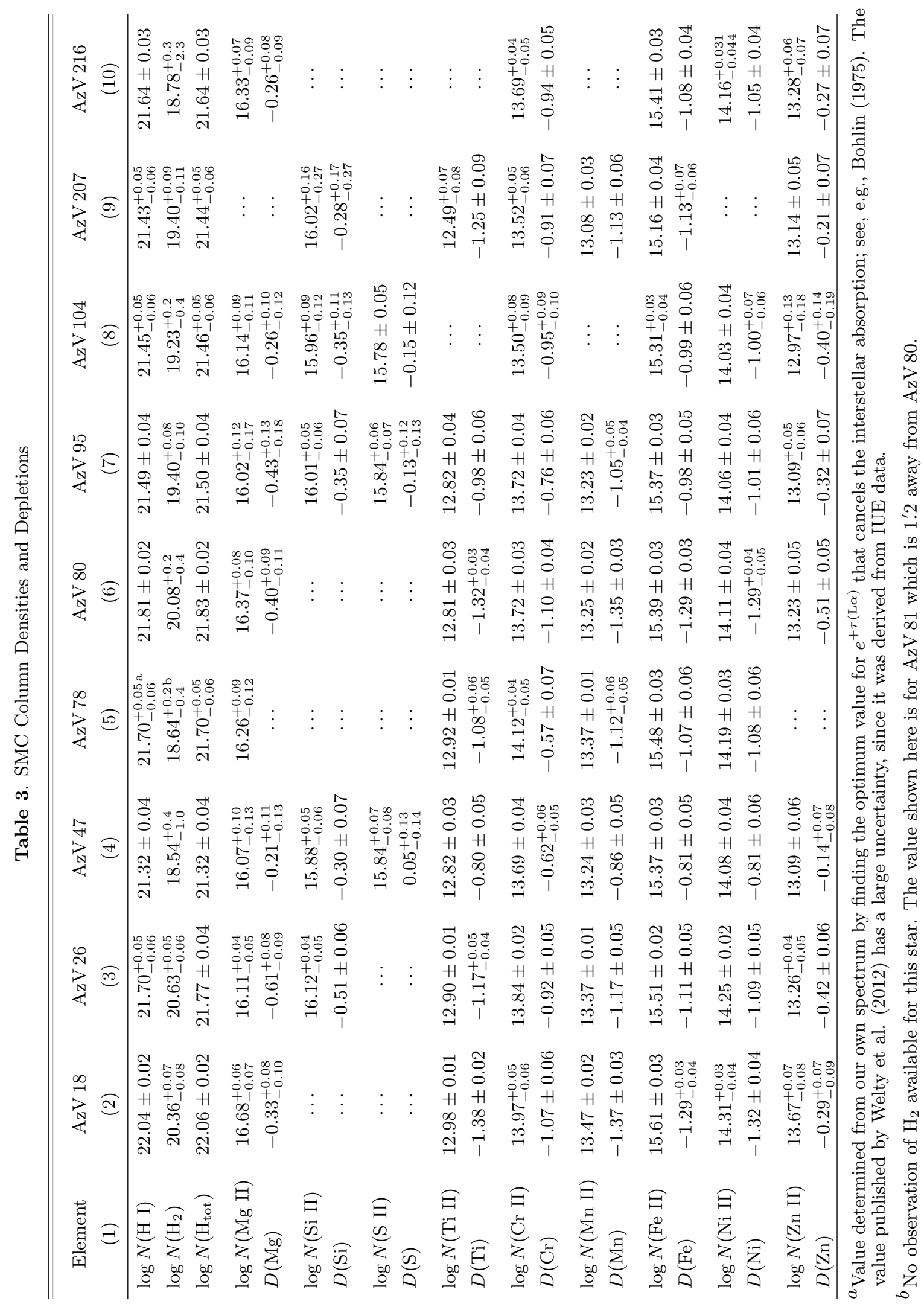




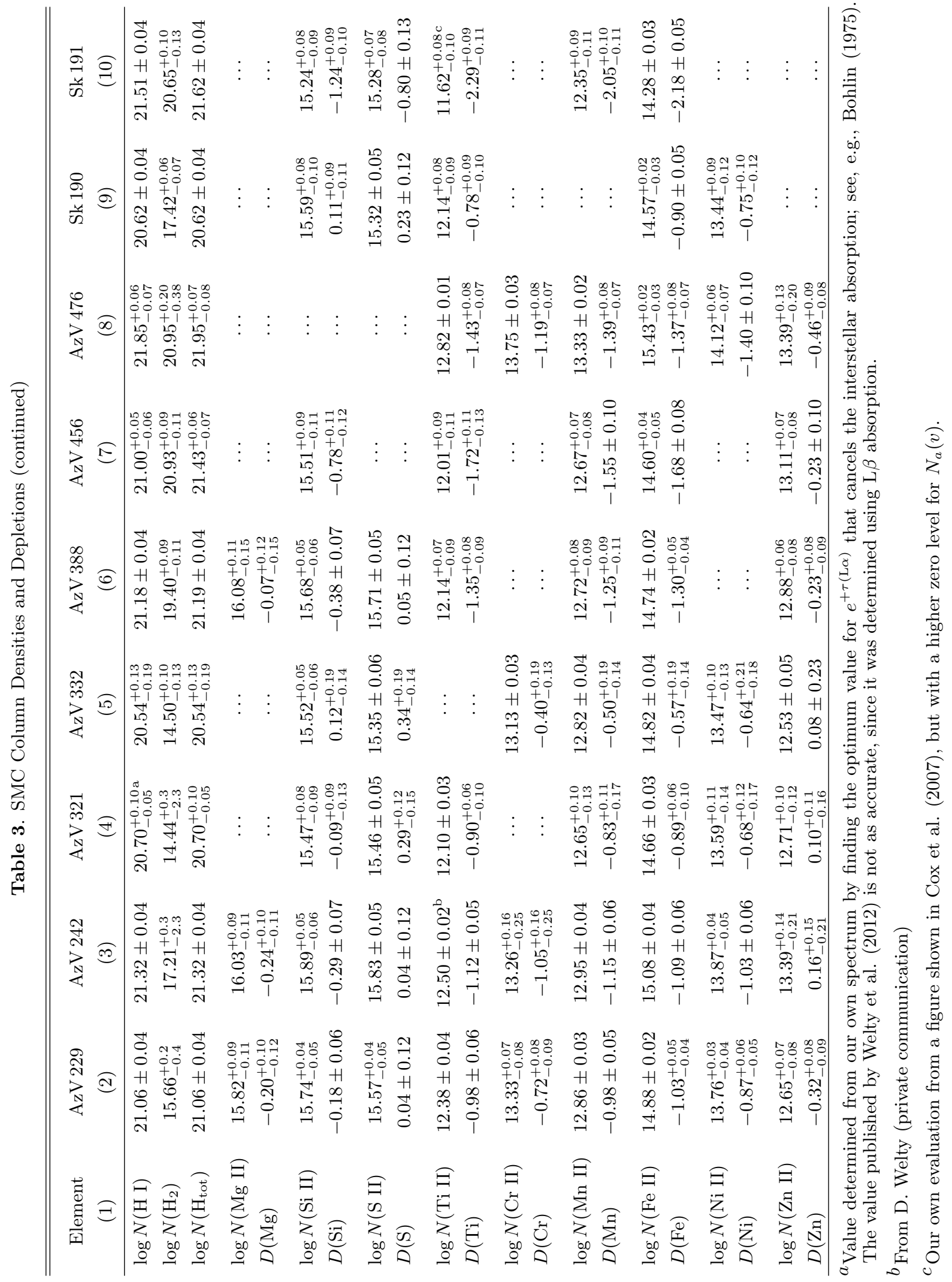




\section{DEPLETION OUTCOMES}

We define the element depletions for the ISM in the SMC in terms of how far the gas-phase concentrations relative to hydrogen are deficient when compared to the stellar abundances that we discussed in Section 5 . We express these depletions $D(X)$ for an element $X$ according to the relation, ${ }^{6}$

$$
D(X)=\log N(X)-\log N\left(\mathrm{H}_{\text {tot }}\right)-\log (X / \mathrm{H})_{\text {stellar }},
$$

where $N\left(\mathrm{H}_{\text {tot }}\right)=N(\mathrm{H} \mathrm{I})+2 N\left(\mathrm{H}_{2}\right)$, and values $(+12)$ for our adopted stellar abundances $\log (\mathrm{X} / \mathrm{H})_{\text {stellar }}$ are listed in Column 5 of Table 2.

The topmost portion of Table 3 shows the logarithms of the $\mathrm{H} \mathrm{I}, \mathrm{H}_{2}$, and $\mathrm{H}_{\text {tot }}$ column densities, most of which were taken from the survey of Welty et al. (2012). The H I column densities were derived from observations of the damping wings of the $\mathrm{L} \alpha$ (and sometimes $\mathrm{L} \beta$ ) absorption features in the spectra of the stars. The outcomes from these measurements include contributions from our Galaxy, which cannot be separated from the SMC components. Hence, an allowance for the foreground Galactic contribution had to be made on the basis of 21-cm measurements in the same directions (Kalberla et al. 2010), using the assumption that the emission was optically thin. Here, the velocity range of Galactic gas was clearly separable from the SMC contributions. These foreground column densities ranged from 3.3 to $7.4 \times 10^{20} \mathrm{~cm}^{-2}$, which constitutes a sizable fraction of the totals for the few SMC sight lines with the lowest values. Welty et al. (2012) determined $N\left(\mathrm{H}_{2}\right)$ from their Voigt profile analyses of Lyman band features arising from the $J=0$ and 1 rotational levels that appeared in FUSE spectra. Here, the Galactic contributions were separable.

The remaining lower part of Table 3 shows in alternating rows the logarithms of the column densities $N(X)$ (in units of $\mathrm{cm}^{-2}$ ) and depletions $D(X)$ for the elements $X$ considered in our study. The uncertainties listed for $D(X)$ were computed from the sums in quadrature for the relative uncertainties in the element and the total hydrogen column densities. We do not include uncertainties in the stellar reference abundances discussed in Section 5 because they are themselves quite uncertain. Moreover, such errors in the reference abundances are irrelevant for the correlations of depletions discussed in Section 7.1 or the differential changes in the individual element depletions with respect to each other (as a function of our generalized depletion parameter), both of which are important themes in this investigation.

\section{DEPLETION RELATIONSHIPS}

Figure 2 shows comparisons of $D(\mathrm{Si})$ and $D(\mathrm{Ti})$ for the corresponding values of $D(\mathrm{Fe})$. This figure shows that the depletions of $\mathrm{Fe}$ and $\mathrm{Ti}$ form a tight, linear sequence. The results for $D(\mathrm{Si})$ show more scatter, but this could be caused by larger uncertainties in the measurements. The solid lines going through the points are best fits based on minimizing the errors in both directions, obtained through the use of the routine FITEXY (Press et al. 2007). If we use the depletion coefficients determined by Jenkins (2009), we can predict how the trends would look for gas in the Milky Way. These relationships are shown by the dashed lines in the figure. It is apparent that the behavior for $D(\mathrm{Si})$ versus $D(\mathrm{Fe})$ in the SMC is very similar to that in the Milky Way, but Ti shows a trend that is less steep in the SMC compared to that of our Galaxy. (Note that similarities or differences in slope represent real propensities for elements to condense into solid form, while vertical displacements could arise simply from inaccuracies in our adopted reference abundances.)

\subsection{Pearson Correlation Coefficients between Elements}

We expect that the depletions of all elements should be correlated with each other. Even so, the strengths of these correlations should be tested to see if the couplings between different combinations of elements show some variation. This is not an easy task, since we must acknowledge that from one element to the next there are very different levels of accuracy for our measurements. These variations will cause the depletions of some elements to show better or worse correlations with those of others. Table 4 shows the strengths of the correlations, expressed in terms of their Pearson correlation coefficients, which are listed in the upper right portion of the upper block of numbers. The numbers of pairs for given combinations of elements are below the diagonal in this upper block.

As pointed out by Jenkins et al. (1986), the comparisons of depletions of elements can have spurious enhancements of their correlations because they share common errors in their denominators, $\log N\left(\mathrm{H}_{\text {tot }}\right)$. Since we have estimates for the errors in $\log N\left(\mathrm{H}_{\text {tot }}\right)$ in our sample, we can correct the correlation coefficients to obtain more realistic values by applying the formulae given by Jenkins et al. (1986, Appendix B). The numbers above the diagonal in the lower block

\footnotetext{
${ }^{6}$ We depart from the notation for a depletion $\left[X_{\text {gas }} / H\right]$ that was adopted by Jenkins $(2009)$ for gas in the Milky Way to avoid a possible misunderstanding about the use of solar abundances as a comparison standard. In our case, we are comparing interstellar abundances in the SMC with those of stars in the SMC.
} 


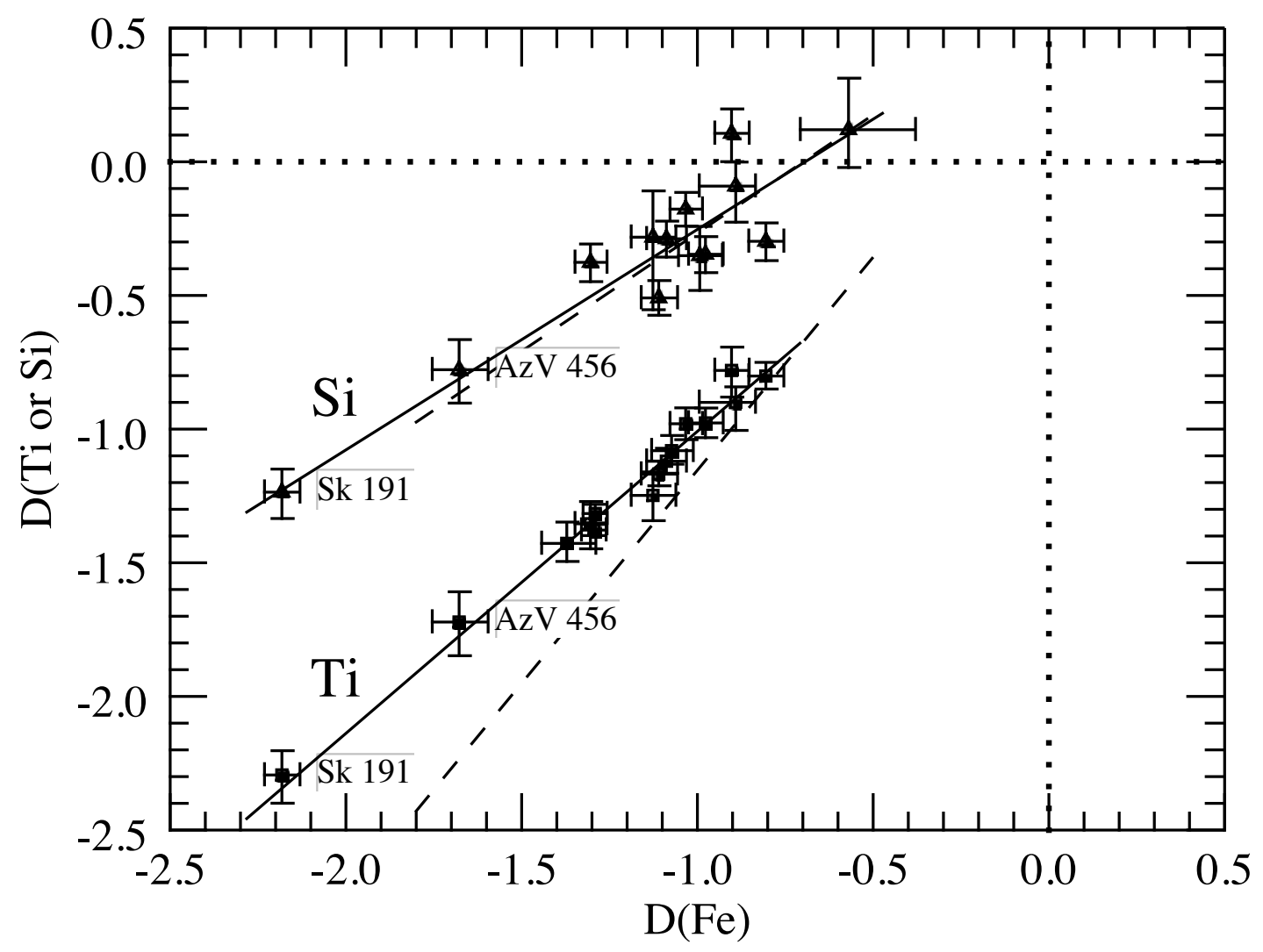

Figure 2. Depletions $D(\mathrm{Si})$ (triangles) and $D(\mathrm{Ti})$ (squares) as a function of $D(\mathrm{Fe})$ for different stars in our survey. The stars $\mathrm{AzV} 456$ and Sk 191 are identified; they represent special cases that are discussed in Section 7.4. The solid lines show the best fits to the data (from minimizing errors in both directions) and the dashed lines show the trends for depletions in the Milky Way using the coefficients given in Jenkins (2009).

of Table 4 show these corrected correlation coefficients. As expected, they are slightly lower than the raw correlation coefficients.

When a correlation of a small sample becomes marginal, it is important to know whether or not it is statistically significant, i.e., what are the chances that over a much larger population the correlation would vanish and the small sample drawn from this population showed a relationship simply by pure chance. A well-established procedure to test the significance of a positive correlation is to evaluate the quantity

$$
t=r\left[\left(1-r^{2}\right) /(n-2)\right]^{-1 / 2},
$$

where $r$ is the observed correlation coefficient for $n$ pairs of samples. The one-tail area of a Student's $t$-distribution applied to the value of $t$ gives the chance that the whole population correlation is less than or equal to zero. The validity of this test relies on the proposition that the population is distributed much like a Gaussian distribution. Since the results for the stars AzV 456 and Sk 191 clearly seem far removed from those of the other stars, we eliminated these two cases when we evaluated the correlations and applied the tests. The values listed below the diagonal of the lower block of numbers show the outcomes for one minus the one-tail area.

It is clear that a majority of combinations show that the positive correlations are real at or above the $95 \%$ level of confidence. Cases with lower confidence may arise simply from larger errors in the measurements or limited ranges over which measurements were possible (e.g., the weakness of the Mg II lines and the saturations of the S II lines limited their reliable ranges of measurement to only about $0.5 \mathrm{dex}$ ). While this may be true, it is worthwhile to investigate whether or not some extreme differences in the apparent correlations could be real, which could be of significance in our interpretations of how the composition of dust particles might vary from one location to the next. For instance, the corrected $r(\mathrm{Mg}, \mathrm{Si})=0.596$ seems to differ from that of $r(\mathrm{Mg}, \mathrm{Fe})=-0.050$. Does this signify that the depletions of $\mathrm{Mg}$ are actually more closely coupled to those of $\mathrm{Si}$ than to those of $\mathrm{Fe}$, or could this be a statistical fluke? If we examine the $95 \%$ confidence intervals for the correlations $\rho$ that one would expect for populations much larger 
than our sample sizes, we conclude that $-0.25 \leq \rho(\mathrm{Mg}, \mathrm{Si}) \leq+0.91$ and $-0.63 \leq \rho(\mathrm{Mg}, \mathrm{Fe}) \leq+0.57$. Since there is a substantial overlap between these two intervals, we are unable to assert at a $95 \%$ level of confidence that the correlations differ from each other, even though in fact they might do so. In contrast to this outcome, another example points toward a real difference in correlations. We identified two cases where we have 15 sample pairs that seemed to differ: $r(\mathrm{Zn}, \mathrm{Fe})=0.438$ and $r(\mathrm{Ti}, \mathrm{Fe})=0.992$, leading to $-0.12 \leq \rho(\mathrm{Zn}, \mathrm{Fe}) \leq+0.75$ and $\rho(\mathrm{Ti}, \mathrm{Fe}) \geq 0.95$, which are clearly incompatible with each other at the $95 \%$ confidence level. While this may be true, we note that $D(\mathrm{Zn})$ does not correlate very well with any of the other elements, which may be just a consequence of the fact that the errors in measuring Zn depletions are large compared to the total range of the outcomes.

Table 4. Pearson Correlation Coefficients ${ }^{\mathrm{a}}$

\begin{tabular}{cccccccccc}
\hline \hline Element & $\mathrm{D}(\mathrm{Mg})$ & $\mathrm{D}(\mathrm{Si})$ & $\mathrm{D}(\mathrm{S})$ & $\mathrm{D}(\mathrm{Ti})$ & $\mathrm{D}(\mathrm{Cr})$ & $\mathrm{D}(\mathrm{Mn})$ & $\mathrm{D}(\mathrm{Fe})$ & $\mathrm{D}(\mathrm{Ni})$ & $\mathrm{D}(\mathrm{Zn})$ \\
\hline $\mathrm{D}(\mathrm{Mg})$ & 10 & 0.633 & 0.723 & 0.054 & 0.251 & 0.201 & 0.025 & 0.470 & 0.463 \\
$\mathrm{D}(\mathrm{Si})$ & 7 & 13 & 0.966 & 0.937 & 0.751 & 0.968 & 0.929 & 0.874 & 0.544 \\
$\mathrm{D}(\mathrm{S})$ & 6 & 10 & 10 & 0.908 & 0.708 & 0.939 & 0.897 & 0.914 & 0.765 \\
$\mathrm{D}(\mathrm{Ti})$ & 8 & 11 & 8 & 15 & 0.849 & 0.985 & 0.992 & 0.955 & 0.372 \\
$\mathrm{D}(\mathrm{Cr})$ & 9 & 8 & 6 & 10 & 13 & 0.894 & 0.868 & 0.845 & 0.484 \\
$\mathrm{D}(\mathrm{Mn})$ & 8 & 11 & 8 & 14 & 11 & 15 & 0.982 & 0.957 & 0.602 \\
$\mathrm{D}(\mathrm{Fe})$ & 10 & 13 & 10 & 15 & 13 & 15 & 18 & 0.934 & 0.481 \\
$\mathrm{D}(\mathrm{Ni})$ & 9 & 9 & 8 & 11 & 12 & 11 & 14 & 14 & 0.702 \\
$\mathrm{D}(\mathrm{Zn})$ & 10 & 11 & 8 & 12 & 12 & 13 & 15 & 12 & 15 \\
& & & & & & & & & \\
$\mathrm{D}(\mathrm{Mg})$ & $\ldots$ & 0.596 & 0.654 & 0.007 & 0.189 & 0.154 & -0.050 & 0.427 & 0.428 \\
$\mathrm{D}(\mathrm{Si})$ & 0.921 & $\ldots$ & 0.965 & 0.936 & 0.709 & 0.968 & 0.927 & 0.858 & 0.487 \\
$\mathrm{D}(\mathrm{S})$ & 0.921 & 0.997 & $\ldots$ & 0.908 & 0.655 & 0.938 & 0.895 & 0.895 & 0.724 \\
$\mathrm{D}(\mathrm{Ti})$ & 0.506 & 0.963 & 0.725 & $\ldots$ & 0.840 & 0.985 & 0.992 & 0.952 & 0.339 \\
$\mathrm{D}(\mathrm{Cr})$ & 0.687 & 0.976 & 0.921 & 0.999 & $\ldots$ & 0.886 & 0.857 & 0.832 & 0.432 \\
$\mathrm{D}(\mathrm{Mn})$ & 0.642 & 0.999 & 0.960 & 1.000 & 1.000 & $\ldots$ & 0.982 & 0.954 & 0.571 \\
$\mathrm{D}(\mathrm{Fe})$ & 0.446 & 0.985 & 0.892 & 1.000 & 1.000 & 1.000 & $\ldots$ & 0.929 & 0.438 \\
$\mathrm{D}(\mathrm{Ni})$ & 0.874 & 0.998 & 0.999 & 1.000 & 1.000 & 1.000 & 1.000 & $\ldots$ & 0.675 \\
$\mathrm{D}(\mathrm{Zn})$ & 0.891 & 0.969 & 0.979 & 0.922 & 0.920 & 0.987 & 0.981 & 0.992 & $\ldots$ \\
\hline
\end{tabular}

${ }^{a}$ Upper half: upper right portion: raw correlation coefficients, lower left portion: numbers of pairs. Lower half: upper right portion: corrected correlation coefficients, lower left portion: the probabilities that the population correlations are greater than zero, given the values of the corrected correlation coefficients and sample sizes (but after omitting data for AzV 456 and Sk 191).

\subsection{Definition of a Generalized Depletion Parameter $F_{*}(\mathrm{SMC})$}

Our principal objective is to describe the element depletions for the SMC gases within a simple framework that follows what Jenkins (2009) did for the local region of our Galaxy. He invented a parameter that he called $F_{*}$ that characterized the general level of depletion for a sight line, and then was able to state that the depletion $\left[X_{\text {gas }} / \mathrm{H}\right]$ of any element $X$ could be represented to good accuracy in the form

$$
\left[X_{\text {gas }} / \mathrm{H}\right]=B_{X}+A_{X}\left(F_{*}-z_{X}\right)
$$

where the coefficients $B_{X}$ and $A_{X}$ were unique for each element $X$, which he obtained from the best fits of the depletion data to the parameter $F_{*}$. While the scale of $F_{*}$ was arbitrary, it was designed such that most sight lines fell within the range $0.0 \leq F_{*} \leq 1.0$. The offset $z_{X}$ for the origin of $F_{*}$ creates a zero covariance in the uncertainties of $B_{X}$ and $A_{X}$ when they are evaluated by the best linear fits to the data. 
The difference in depletion behaviors between Ti and Fe for the Milky Way and the SMC shown in Fig. 2 indicates that a definition of $F_{*}$ based on the depletions of different elements in the Milky Way is no longer appropriate. For this reason, we must now define a new measure of $F_{*}$ that can be applied to gas in the SMC, which we will designate as $F_{*}(\mathrm{SMC})$. For the $\mathrm{SMC}$, we do not have as broad a selection of elements and target stars to establish in an independent and self-consistent manner a depletion scale as Jenkins (2009) was able to do. Instead, we focus chiefly on the depletions of Fe, which was measured for every star in our SMC survey. At the same time, it is desirable to design this new depletion parameter such that it is similar to the one defined earlier for the Milky Way, which we now call $F_{*}(\mathrm{MW})$. In order to do so, we have chosen to link the two scales through the depletion of Fe, which through a simple transformation of Eq. 3 leads us to our proposed definition for any particular sightline,

$$
F_{*}(\mathrm{SMC})=\frac{D(\mathrm{Fe})-B_{\mathrm{Fe}}}{A_{\mathrm{Fe}}}+z_{\mathrm{Fe}},
$$

where we adopt the Milky Way values $B_{\mathrm{Fe}}=-1.51, A_{\mathrm{Fe}}=-1.28$, and $z_{\mathrm{Fe}}=0.437$.

While the measurement uncertainties of $D(\mathrm{Fe})$ are small, we can increase the accuracy of $F_{*}(\mathrm{SMC})$ if we also consider the depletions of two other elements, $\mathrm{Ti}$ and $\mathrm{Mn}$, which are also well determined. After evaluating the best fits of the depletions of these two elements relative to that of Fe [e.g., see Fig. 2 that shows $D(\mathrm{Ti})$ vs $D(\mathrm{Fe})$ ], for each individual sight line we use these best-fit relations to transform $D(\mathrm{Ti})$ and $D(\mathrm{Mn})$ to their equivalent Fe depletions and then evaluate a weighted average to optimize the equivalent value of the Fe depletion for determining $F_{*}(\mathrm{SMC})$ through the application of Eq. 4. As with the measurements of column densities discussed in Section 4, the weights are proportional to $1 / \sigma_{i}^{2}$ and the final uncertainty in $F_{*}(\mathrm{SMC})$ equals $\left(\sum_{i} \sigma_{i}^{-2}\right)^{-0.5}$. Table 5 lists the outcomes for all of the stars in our survey.

Table 5. $F_{*}(\mathrm{SMC})$

\begin{tabular}{cr}
\hline \hline Star & \multicolumn{1}{c}{$F_{*}(\mathrm{SMC})$} \\
\hline AzV 18 & $0.29 \pm 0.01$ \\
$\mathrm{AzV} 26$ & $0.14 \pm 0.02$ \\
$\mathrm{AzV} 47$ & $-0.11 \pm 0.03$ \\
$\mathrm{AzV} 78$ & $0.09 \pm 0.03$ \\
$\mathrm{AzV} 80$ & $0.27 \pm 0.02$ \\
$\mathrm{AzV} 95$ & $0.02 \pm 0.02$ \\
$\mathrm{AzV} 104$ & $0.03 \pm 0.05$ \\
$\mathrm{AzV} 207$ & $0.14 \pm 0.03$ \\
$\mathrm{AzV} 216$ & $0.10 \pm 0.03$ \\
$\mathrm{AzV} 229$ & $0.03 \pm 0.02$ \\
$\mathrm{AzV} 242$ & $0.11 \pm 0.02$ \\
$\mathrm{AzV} 321$ & $-0.05 \pm 0.04$ \\
$\mathrm{AzV} 332$ & $-0.35 \pm 0.10$ \\
$\mathrm{AzV} 388$ & $0.27 \pm 0.03$ \\
$\mathrm{AzV} 456$ & $0.53 \pm 0.04$ \\
$\mathrm{AzV} 476$ & $0.33 \pm 0.03$ \\
Sk 190 & $-0.06 \pm 0.03$ \\
$\mathrm{Sk} 191$ & $0.94 \pm 0.03$ \\
\hline
\end{tabular}




\subsection{How Element Depletions Scale with $F_{*}(\mathrm{SMC})$}

For each element $X$, we determined the best fit of the depletions to the $F_{*}(\mathrm{SMC})$ parameter, again by minimizing errors in both the dependent and independent quantities using the linear fit routine FITEXY. The outcomes for $A_{X}$, $B_{X}$, and $z_{X}$ are shown in the right-hand half of Table 6 , which can be compared to the same set of coefficients that apply to the local $F_{*}(\mathrm{MW})$ that are listed in the left-hand side of the table. Figure 3 shows plots that exhibit the depletion measurements versus $F_{*}(\mathrm{SMC})$ along with the fits constructed from Eq. 3. It is important to emphasize that any deviations in the stellar reference abundances from our adopted $[X / \mathrm{H}]=-0.65$, as indicated by the (somewhat uncertain) numbers listed in Column 6 of Table 2, will be reflected in the form of vertical offsets in the trends, as defined by the $B_{X}$ parameters. The $A_{X}$ slope parameters are immune to errors in these reference abundances.

Table 6. Fit Coefficients for $F_{*}(\mathrm{MW})$ and $F_{*}(\mathrm{SMC})$

\begin{tabular}{|c|c|c|c|c|c|c|c|c|c|}
\hline \multirow[b]{2}{*}{$X$} & \multicolumn{3}{|c|}{ Milky Way (MW) } & \multicolumn{6}{|c|}{ Small Magellanic Cloud (SMC) } \\
\hline & $A_{X}$ & $B_{X}$ & $z_{X}$ & $A_{X}$ & $B_{X}{ }^{\mathrm{a}}$ & $z_{X}$ & $\chi^{2}$ & $\nu$ & $P^{\mathrm{b}}$ \\
\hline $\mathrm{Mg}$ & $-1.00 \pm 0.04$ & $-0.80 \pm 0.02$ & 0.531 & $-0.25 \pm 0.26$ & $-0.33 \pm 0.03$ & 0.162 & 22.1 & 8 & 0.005 \\
\hline $\mathrm{Si}$ & $-1.14 \pm 0.06$ & $-0.57 \pm 0.03$ & 0.305 & $-1.05 \pm 0.09$ & $-0.36 \pm 0.02$ & 0.129 & 26.7 & 11 & 0.005 \\
\hline $\mathrm{S}$ & $-0.88 \pm 0.28$ & $-0.09 \pm 0.04$ & 0.290 & $-0.87 \pm 0.14$ & $-0.02 \pm 0.04$ & 0.106 & 11.1 & 8 & 0.196 \\
\hline $\mathrm{Ti}$ & $-2.05 \pm 0.06$ & $-1.96 \pm 0.03$ & 0.430 & $-1.45 \pm 0.09$ & $-1.23 \pm 0.02$ & 0.189 & 2.3 & 13 & 0.999 \\
\hline $\mathrm{Cr}$ & $-1.45 \pm 0.06$ & $-1.51 \pm 0.05$ & 0.470 & $-1.33 \pm 0.16$ & $-0.93 \pm 0.02$ & 0.155 & 18.7 & 11 & 0.066 \\
\hline Mn & $-0.86 \pm 0.04$ & $-1.35 \pm 0.03$ & 0.520 & $-1.20 \pm 0.09$ & $-1.24 \pm 0.02$ & 0.196 & 5.1 & 13 & 0.973 \\
\hline $\mathrm{Fe}$ & $-1.28 \pm 0.04$ & $-1.51 \pm 0.03$ & 0.437 & $-1.28 \pm 0.07$ & $-1.18 \pm 0.02$ & 0.181 & 1.8 & 16 & 1.000 \\
\hline $\mathrm{Ni}$ & $-1.49 \pm 0.06$ & $-1.83 \pm 0.04$ & 0.599 & $-1.41 \pm 0.14$ & $-1.11 \pm 0.02$ & 0.141 & 7.0 & 12 & 0.857 \\
\hline $\mathrm{Zn}$ & $-0.61 \pm 0.07$ & $-0.28 \pm 0.05$ & 0.555 & $-0.51 \pm 0.14$ & $-0.31 \pm 0.02$ & 0.168 & 44.8 & 13 & 0.000 \\
\hline
\end{tabular}

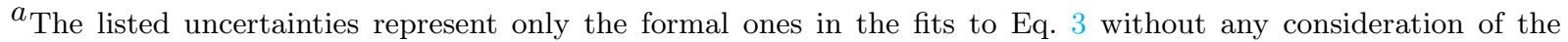
possible real deviations from the stellar reference abundances listed in Column 5 of Table 2.

$b$ The probability of obtaining a worse fit to the linear trend, given the $\chi^{2}$ values and their respective degrees of freedom $\nu$, which are driven by our estimates for the individual errors in both directions. A low value indicates that either we have underestimated the errors or that real depletions deviate from a simple linear relationship with $F_{*}(\mathrm{SMC})$.

Figure 3 shows that the fits for $\mathrm{Mg}$, Ti, and Mn for the Milky Way (dashed lines) differ from those for the SMC. While there seems to be a vertical offset between the two for Zn, one could discount this as arising either from an error in the adopted SMC stellar abundance for $\mathrm{Zn}$ or the more complex nucleosynthesis origins for this element (note that the slopes $A_{\mathrm{Zn}}$ are nearly identical to each other to within their uncertainties). The remaining elements, $\mathrm{Si}, \mathrm{S}, \mathrm{Cr}$, and $\mathrm{Ni}$, seem to have depletion sequences in the SMC that are very similar to those seen in the Milky Way (the two relationships for Fe are identical by construction). Vladilo (2002) found that Mn seemed to exhibit stronger depletions in the SMC relative to the standard depletion patterns for other elements, which is consistent with our finding that $A_{\mathrm{Mn}}$ for the SMC indicates a more negative slope than its counterpart for the Milky Way. The weaker Si depletions relative to the Galactic trends are not duplicated by our findings, where we determined that $A_{\mathrm{Si}}$ for the SMC is about identical to that for the Milky Way. 

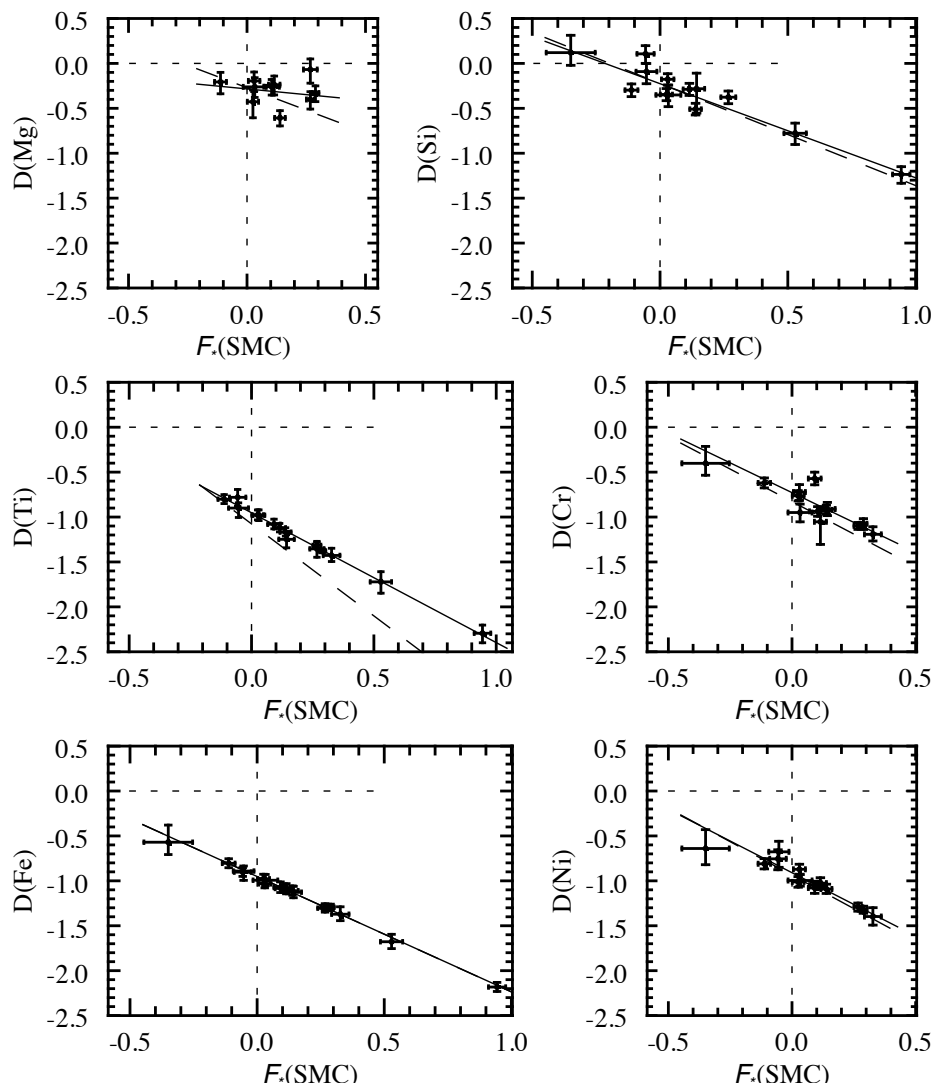
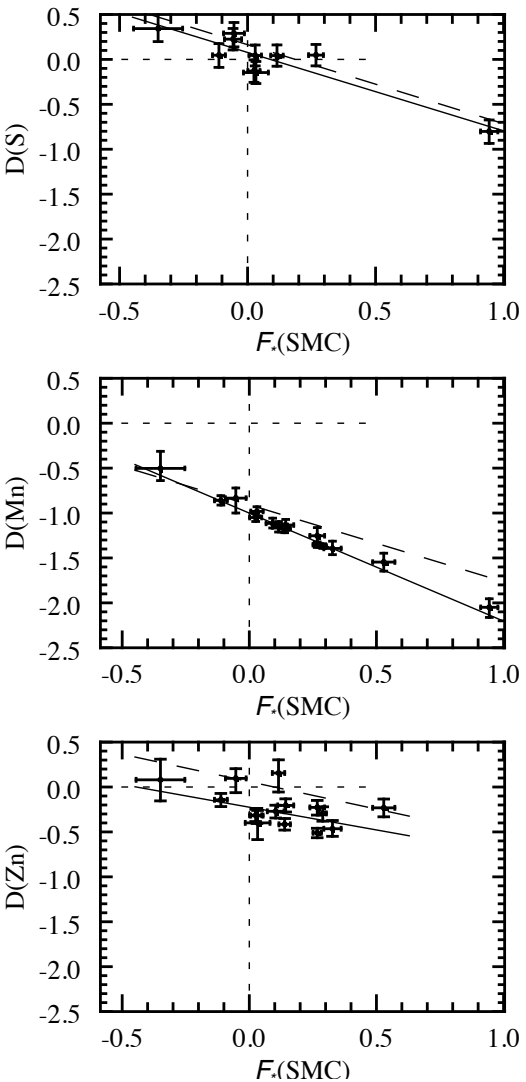

Figure 3. Element depletions vs. $F_{*}(\mathrm{SMC})$. Solid lines show the best fits, with the coefficients given in Table 6 . Dashed lines represent fits that were obtained for our Galaxy. By design, the fits for $D(\mathrm{Fe})$ for our Galaxy and the SMC coincide with each other. The points located at $F_{*}(\mathrm{SMC}) \approx 0.5$ and 0.9 represent $\mathrm{AzV} 456$ and $\mathrm{Sk} 191$, respectively.

\subsection{The Special Cases of $A z V 456$ and Sk 191}

There are two stars that exhibited depletion outcomes that appeared to be markedly stronger than those for other stars in the survey. One of them, AzV 456, is the most highly reddened star in our sample (see Table 1), exhibits relatively strong absorptions by diffuse interstellar bands (Welty et al. 2006 ; Cox et al. 2007), shows absorption features arising from $\mathrm{C}_{2}, \mathrm{C}_{3}, \mathrm{CN}$ and $\mathrm{CH}$ (Welty et al. 2013), has a large fraction of hydrogen in molecular form (see Table 3) and, unlike most other stars in the SMC, exhibits a UV extinction curve that is similar to that of our Galaxy (Gordon \& Clayton 1998 ; Cartledge et al. 2005 ; Cox et al. 2007). From their analysis of the rotational excitations of $\mathrm{C}_{2}$ and $\mathrm{H}_{2}$, Welty et al. (2013) found a low kinetic temperature and a moderately high density for the absorbing cloud in front of $\mathrm{AzV} 456$, although the thermal pressure derived from the C I fine-structure excitation seems lower than $n k T$ indicated by the molecules (Welty et al. 2016). For these reasons, it is no surprise that this star is well removed from the others in the depletion plots shown in Figs. 2 and 3. We note that that its depletions for different elements seem to follow extrapolations of the sequences with $F_{*}(\mathrm{SMC})$ for the other stars in our survey.

The other star, Sk 191, shows even more depletion than AzV 456. This star is located in the southeastern "wing" of the SMC, but unlike AzV 456 the sight line toward this star does not exhibit an especially large reddening or high $\mathrm{H}_{2}$ fraction. This star is the furthest removed from the others and is close to the Magellanic Bridge (MB), a region containing gas and stars that spans the SMC and LMC. Dynamical simulations by Gardiner \& Noguchi (1996) and Hammer et al. (2015) suggest that the MB was created by a tidal interaction when the Large and Small Magellanic Clouds collided some 200-300 Myr ago. Several stars embedded in the MB midway between the SMC and LMC show abundances even lower than those in the SMC (Rolleston et al. 1999 ; Lee et al. 2005 ; Dufton et al. 2008), and the results of Lehner et al. (2001); (2008) indicate that the same holds for gas in this region.

A legitimate question is whether or not the environment in front of Sk 191 likewise has an intrinsically lower total abundance of heavy elements compared to the material in front of the other stars in the SMC (Welty \& Crowther 2010). A revealing indication is that stars closest to Sk 191 investigated by Lee et al. (2005) seem to have abundances 
that more closely match those of stars within the SMC proper, and the same holds for Sk 191 itself (Trundle et al. 2004). Moreover, the spectrum of Sk 191 is one of the very few cases where we have a marginal detection of interstellar O I absorption at $1356 \AA$ that lines up perfectly at the correct velocity. Our measurement of the equivalent width of this weak feature is $9.1 \pm 7.6 \mathrm{~m} \AA$, which leads to a value $\log N(\mathrm{O} \mathrm{I})=17.68$. If one imagines that the depletion of $\mathrm{O}$ is almost zero, we would expect to find $\log N(\mathrm{O} \mathrm{I})=17.66$ for $\log N(\mathrm{H})=21.62$ and $[\mathrm{M} / \mathrm{H}]$ equal our adopted value for the SMC of -0.65 . If this detection is real and its magnitude is approximately correct, we would conclude that the intrinsic abundances of the gas in front of this star should not be appreciably lower than elsewhere in the SMC. In short, various considerations favor the idea that extraordinarily strong depletions onto grains are responsible for the low abundances toward this star.

\subsection{Sulfur: A Troublesome Element}

The title of this subsection is identical to one that appeared in Jenkins (2009). In studies of S II absorption toward stars in the Milky Way, most of the sight lines that satisfied a criterion that $\log N\left(\mathrm{H}_{\text {tot }}\right)>19.5$ (to eliminate the need for ionization corrections) also had enough ionized sulfur to make all three lines of the triplet completely saturated and hence unusable. Even for the few cases where acceptable results for S could be obtained, there was also the concern that some of the S II could reside within the H II regions surrounding the target star, since the ionization potential of $\mathrm{S}^{+}$is high $(23.4 \mathrm{eV})$. There were a number of examples in the study by Jenkins (2009) that indicated the presence of apparent super-solar abundances of $\mathrm{S}$ if one assumed that all of the $\mathrm{S}$ was in an $\mathrm{H} \mathrm{I}$ region.

For the SMC, the overall lower metallicity of the gas gives us a larger margin to observe absorption features that are not totally saturated and yet have enough total gas in the sight line so that ionization corrections and the contributions from H II regions are not substantial. The stellar temperatures of most of the stars in our survey are high enough that appreciable fractional concentrations of $\mathrm{S}^{+}$should only be expected in the outer few percent of a line through the H II region (Sarazin 1977 ; Evans \& Dopita 1985 ; Evans 1991). However, it is possible that some of our sight lines may accidentally penetrate the H II regions of foreground B-type stars, where there are far fewer photons that can convert $\mathrm{S}$ to the doubly ionized state because their fluxes are strongly attenuated at energies that are above the ionization potential of neutral He at $24.6 \mathrm{eV}$ (Vallerga \& Welsh 1995 ; Cassinelli et al. 1996), which nearly coincides with that of $\mathrm{S}^{+}$. However, such stars can still fully ionize the accompanying hydrogen.

The results in Table 3 indicate that for three of our stars, AzV 321, AzV 332, and Sk 190, the interstellar S abundances appear to be approximately twice as large as the stellar abundance standard that we adopted, i.e., $D(\mathrm{~S}) \approx+0.3$. Values of $N\left(\mathrm{H}_{\text {tot }}\right)$ for these three stars are lower than for all of the others in our sample, they have negative values for $F_{*}(\mathrm{SMC})$, and there is weak evidence that both $D(\mathrm{Si})$ and $D(\mathrm{Zn})$ may be positive, but by amounts that are not as significant as our findings for S. Deviations in the measured stellar abundances of $\mathrm{S}$ from our adopted reference standard appear to be small, as indicated by the entry for this element in Column 6 of Table 2. Thus, as with the Milky Way, the results for S in the SMC present the puzzling outcomes that interstellar S abundances occasionally exceed those for young stars in the same general environment. However, the possibility that the accompanying $\mathrm{Si}$ and $\mathrm{Zn}$ might also have higher than expected concentrations may indicate that local abundance anomalies may be responsible for these deviations (the separation in the sky for AzV 321 and AzV 332 is only 155").

\section{BUILDUP OF ELEMENTS IN DUST GRAINS}

If we consider the amount of any particular element in dust, relative to the amount of hydrogen,

$$
\left(X_{\text {dust }} / \mathrm{H}\right)=(X / \mathrm{H})_{\text {stellar }}\left(1-10^{D_{X}}\right),
$$

combine it with Eq. 3, and differentiate with respect to $F_{*}(\mathrm{SMC})$, we can monitor the rates of condensation of elements onto grains as $F_{*}(\mathrm{SMC})$ increases using the equation

$$
\begin{aligned}
\frac{d\left(X_{\text {dust }} / \mathrm{H}\right)}{d F_{*}(\mathrm{SMC})} & =-(\ln 10)(X / \mathrm{H})_{\text {stellar }} A_{X} 10^{A_{X}\left(F_{*}(\mathrm{SMC})-z_{X}\right)+B_{X}} \\
& =-(\ln 10) A_{X}\left(X_{\text {gas }} / \mathrm{H}\right)_{F_{*}(\mathrm{SMC})}
\end{aligned}
$$

This differential analysis has the advantage of being independent of the adopted stellar abundances and their associated uncertainties. It requires a knowledge of only the slope parameter $A_{X}$ and the (linear) expression for the relative abundance of the element $X$ with respect to $\mathrm{H}_{\text {tot }}$ at a particular value of $F_{*}(\mathrm{SMC})$. Figure 4 shows the trends for the consumptions of atoms in both the SMC and the Milky Way. 

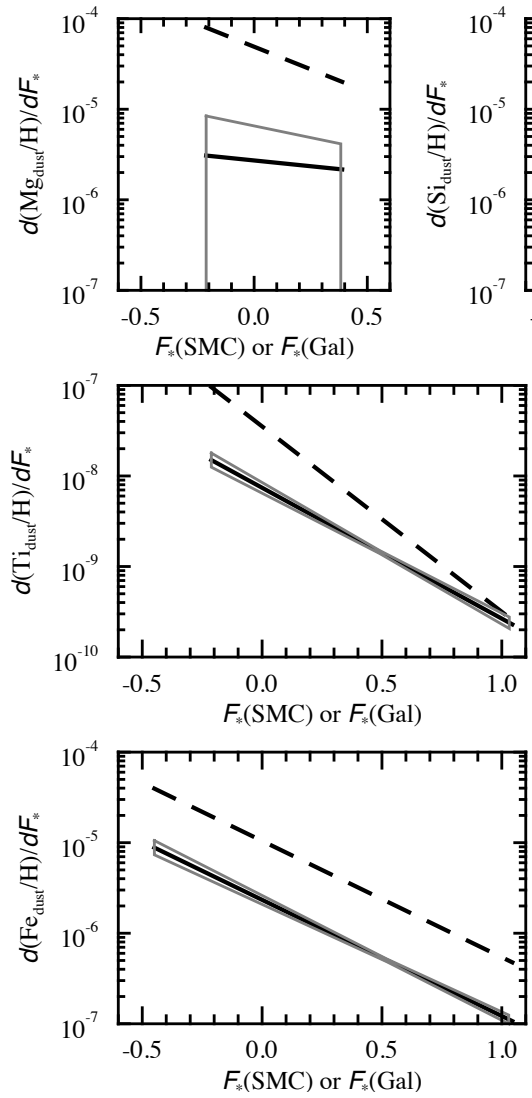
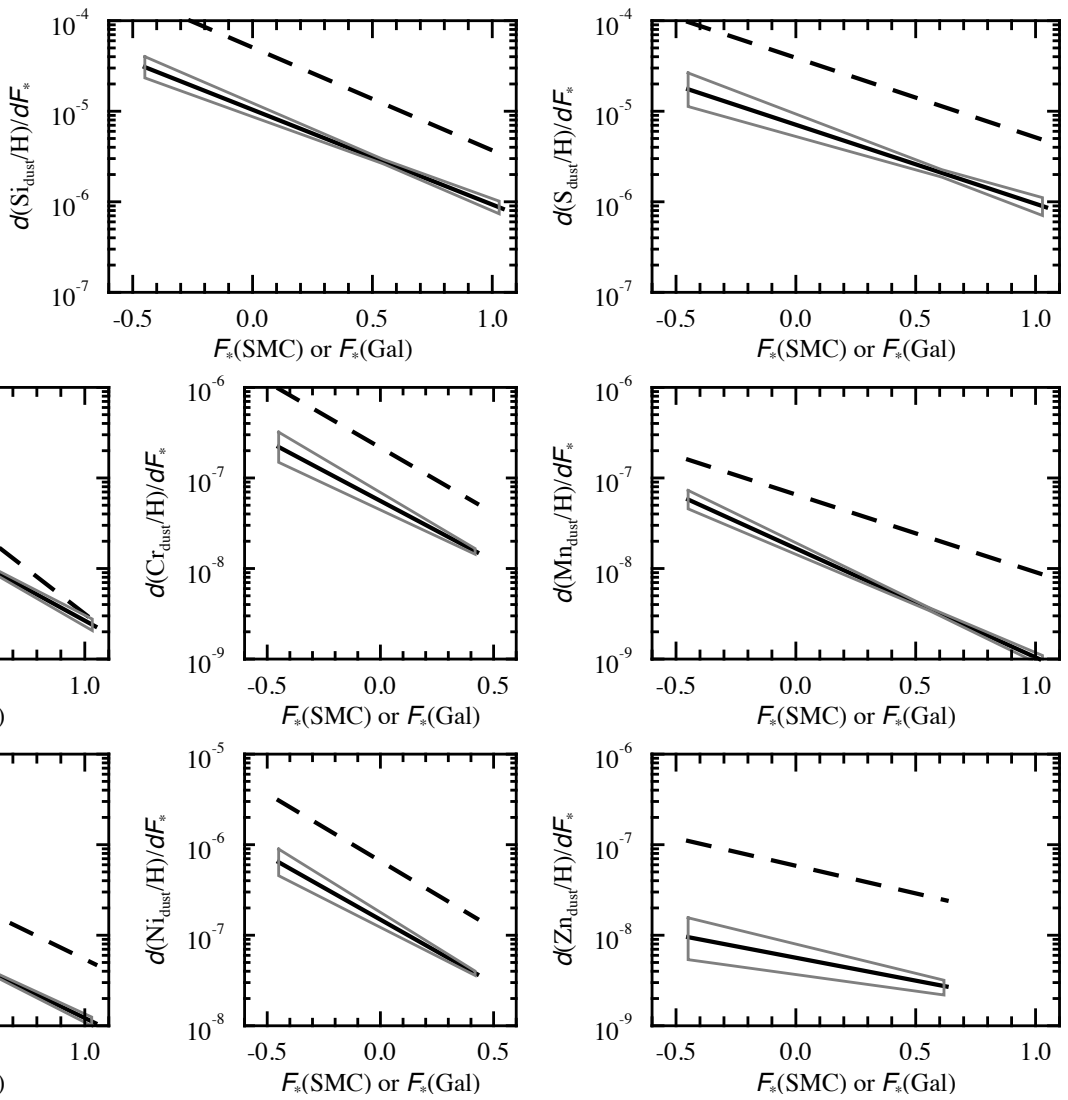

Figure 4. solid lines: Differential consumptions of elements by number (relative to hydrogen) by dust grains for small changes in $F_{*}(\mathrm{SMC})$ plotted as a function of $F_{*}(\mathrm{SMC})$. The surrounding uncertainty envelopes representing the $\pm 1 \sigma$ deviations in $A_{X}$ and $B_{X}$ are shown by light lines. Dashed lines: The same for dust consumption in the Milky Way (as a function of $F_{*}(\mathrm{MW})$ ). The large vertical displacements between the two lines reflect that fact that the SMC has a 0.65 dex lower metallicity than our Galaxy, so changes in depletion in the SMC result in fewer atoms condensing onto the grains.

\section{DISCUSSION}

\subsection{General Remarks}

The depletion trends that we presented in Section 7.3 indicate that most elements deplete together in a manner similar to that of the Milky Way (e.g., compare the solid and dashed lines in Fig. 3). Two exceptions that we found to be significant are the elements $\mathrm{Ti}$ and $\mathrm{Mn}$, as indicated by our finding that the outcomes for $A_{\mathrm{Ti}}$ and $A_{\mathrm{Mn}}$ in the SMC differ from their counterparts in the Milky Way by more than their uncertainties (see Table 6). (Apparent differences in the listed $B_{X}$ values are of no fundamental significance, since they could be influenced by possible deviations in the true total abundances from our adopted reference abundances and also the differences in the $z_{X}$ values.) Mg does not appear to deplete as rapidly in the SMC as it does in the Milky Way, but we have fewer measurements (because they were more difficult to perform), and the scatter in the outcomes is large, as indicated by the low value for $P$ in the last column of Table 6. The scatter in the Zn depletions is large, although the worst fluctuations occur for measurements with the largest uncertainties.

\subsection{A Comparison with the SMC Depletions of Tchernyshyov et al. (2015)}

Tchernyshyov et al. (2015) have conducted a depletion study of gas within both the SMC and LMC, based on UV spectroscopic data from FUSE and the COS on the HST. Their objective was similar to ours: they made comparisons of depletions of different elements and derived relationships using the same basic formalism as ours, except that they did not use an offset $z_{X}$ to eliminate error covariances in the slope and overall depletion parameters. For elements and target stars that are common to our two surveys, the results for column densities agree with each other to within about 0.1 dex, except for Si II and $\mathrm{Zn}$ II toward $\mathrm{AzV}$ 456. Their definition for their generalized depletion strength, $F^{*}$, differs slightly from our $F_{*}(\mathrm{SMC})$. If we anchor the two systems to the depletion trends of Fe, an element with the best measurement accuracies in both surveys, we derive a relationship $F^{*}=1.13 F_{*}(\mathrm{SMC})+0.334$. To convert from 
their parameters ${ }^{7} A_{X}(\mathrm{~T} 15)$ and $\delta(X)_{0}(\mathrm{~T} 15)$ to ours, we apply the transformations

$$
A_{X}=1.13 A_{X}(\mathrm{~T} 15)
$$

and

$$
B_{X}=A_{X}(\mathrm{~T} 15)\left(1.13 z_{X}+0.334\right)+\delta(X)_{0}(\mathrm{~T} 15) .
$$

Columns $2-5$ of Table 7 show a comparison of their findings to ours, after converting their parameters to our system through Eqs. 7 and 8. Our values and theirs overlap to within the uncertainty ranges of both determinations, except for $B_{\mathrm{Si}}$ and $A_{\mathrm{Zn}}$, where for each case our lower bound approximately matched their upper bound. This table also shows their outcomes for the element $\mathrm{P}$, which was not included in our survey.

\begin{tabular}{|c|c|c|c|c|c|c|}
\hline \multirow[b]{2}{*}{$\begin{array}{l}\text { Element } \\
\text { (1) }\end{array}$} & \multicolumn{2}{|c|}{$\begin{array}{c}\text { SMC Values from } \\
\text { Tchernyshyov et al. (2015) } \\
\text { (using Eqs. } 7 \text { and } 8 \text { ) }\end{array}$} & \multicolumn{2}{|c|}{$\begin{array}{l}\text { SMC Values from } \\
\text { this Paper }^{\mathrm{b}}\end{array}$} & \multicolumn{2}{|c|}{$\begin{array}{l}\text { DLA Values from } \\
\text { De Cia et al. (2016) } \\
\text { (Using Eqs. } 9 \text { and 10) }\end{array}$} \\
\hline & $\begin{array}{l}A_{X} \\
(2)\end{array}$ & $\begin{array}{r}B_{X} \\
(3)\end{array}$ & $\begin{array}{r}A_{X} \\
(4)\end{array}$ & $\begin{array}{l}B_{X} \\
(5)\end{array}$ & $\begin{array}{l}A_{X} \\
(6)\end{array}$ & $\begin{array}{r}B_{X} \\
(7)\end{array}$ \\
\hline $\mathrm{O}$ & $\cdots$ & $\cdots$ & $\cdots$ & $\cdots$ & $-0.15 \pm 0.04$ & $-0.15 \pm 0.13^{\mathrm{c}}$ \\
\hline $\mathrm{Mg}$ & $\cdots$ & $\cdots$ & $-0.25 \pm 0.26$ & $-0.33 \pm 0.03$ & $-0.62 \pm 0.05$ & $-0.58 \pm 0.07$ \\
\hline $\mathrm{Si}$ & $-1.16 \pm 0.17$ & $-0.50 \pm 0.11$ & $-1.05 \pm 0.09$ & $-0.36 \pm 0.02$ & $-0.64 \pm 0.06$ & $-0.58 \pm 0.06$ \\
\hline $\mathrm{P}$ & $-0.94 \pm 0.08$ & $-0.32 \pm 0.05^{\mathrm{c}, \mathrm{d}}$ & $\cdots$ & $\cdots$ & $-0.10 \pm 0.07$ & $-0.08 \pm 0.09^{\mathrm{c}}$ \\
\hline $\mathrm{S}$ & $\cdots$ & $\ldots$ & $-0.87 \pm 0.14$ & $-0.02 \pm 0.04$ & $-0.28 \pm 0.08$ & $-0.28 \pm 0.07$ \\
\hline $\mathrm{Cr}$ & $-1.10 \pm 0.12$ & $-0.85 \pm 0.08$ & $-1.33 \pm 0.16$ & $-0.93 \pm 0.02$ & $-1.34 \pm 0.10$ & $-1.04 \pm 0.05$ \\
\hline Mn & $\cdots$ & & $-1.20 \pm 0.09$ & $-1.24 \pm 0.02$ & $-0.97 \pm 0.04$ & $-0.86 \pm 0.05$ \\
\hline $\mathrm{Fe}^{\mathrm{e}}$ & $-1.28 \pm 0.08$ & $-1.18 \pm 0.06$ & $-1.28 \pm 0.07$ & $-1.18 \pm 0.02$ & $-1.28 \pm 0.04$ & $-1.18 \pm 0.05$ \\
\hline $\mathrm{Zn}$ & $-0.70 \pm 0.07$ & $-0.36 \pm 0.07$ & $-0.51 \pm 0.14$ & $-0.31 \pm 0.02$ & $-0.27 \pm 0.03$ & $-0.25 \pm 0.03$ \\
\hline
\end{tabular}

Table 7. Parameter Comparisons ${ }^{\mathrm{a}}$

$a_{\text {Eqs. }}$, 8, 9, and 10 are used to convert the coefficients for the relationships found by the two other investigators into our $A_{X}$ and $B_{X}$ parameters that are linked to $F_{*}(\mathrm{SMC})$.

${ }^{b}$ Copied from Table 6 for ease of comparison.

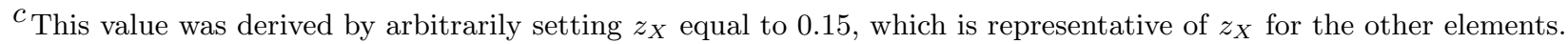

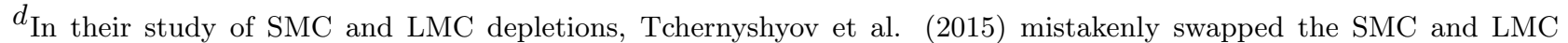
reference abundances for $\mathrm{P}$. We have applied an upward correction for $B_{\mathrm{P}}$ of 0.4 dex to overcome this error.

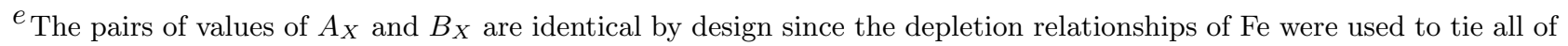
the parameter systems together.

\subsection{Relating our Results to DLA Depletions}

While our investigation is intended to guide future interpretations of gas-phase abundances measured for DLA and sub-DLA systems, it is also useful to look back on some previous investigations of these systems in order to learn how well, or how poorly, our depletion patterns match them. We discussed in Section 2 some examples of puzzling deviations from the Galactic depletion patterns. The few departures that we see between the SMC and the Milky Way indicate that some depletions do not behave in a universal fashion for all possible combinations of metal-bearing systems.

Ledoux et al. (2002) examined the trends of $\mathrm{Si}, \mathrm{Ti}, \mathrm{Cr}$, and $\mathrm{Mn}$ abundances relative to $\mathrm{Fe}$ as a function of [Zn/Fe]. It is generally accepted that the depletions of $\mathrm{Zn}$ are weak and those of Fe are strong, so their depletion ratio can serve as a proxy for the relative generalized depletions. They found that $[\mathrm{Si} / \mathrm{Fe}]$ increases with increasing $[\mathrm{Zn} / \mathrm{Fe}]$,

7 We append (T15) to these parameters to avoid confusion with our parameters. 
which is qualitatively consistent with our conclusion that in the SMC $A_{\mathrm{Si}}>A_{\mathrm{Fe}}$ (i.e., Si depletes less rapidly than Fe; note that in the Milky Way the difference between the two may be slightly less). Their values of [Cr/Fe] seem to be independent of $[\mathrm{Zn} / \mathrm{Fe}]$, which agrees with our conclusion that $A_{\mathrm{Cr}} \approx A_{\mathrm{Fe}}$ in the $\mathrm{SMC}$ ( $\mathrm{Cr}$ depletes more rapidly than Fe in the Milky Way). However, they find substantial increases in $[\mathrm{Mn} / \mathrm{Fe}]$ when $[\mathrm{Zn} / \mathrm{Fe}]$ becomes larger, which seems inconsistent with our determination that $A_{\mathrm{Mn}}$ is not much different from $A_{\mathrm{Fe}}$ in the SMC. The trend for Mn seems more consistent with the pattern in the Milky Way, where $A_{\mathrm{Mn}}$ shows a substantially shallower slope than that for $A_{\mathrm{Fe}}$.

A recent, more comprehensive investigation of DLA element depletions has been carried out by De Cia et al. (2016). They too used $[\mathrm{Zn} / \mathrm{Fe}]$ as an indicator for depletion, but they also made use of the finding that $[\mathrm{Zn} / \mathrm{Fe}]$ is correlated with $[\mathrm{Zn} / \mathrm{H}]$ (Wolfe et al. 2005), which serves as an approximate indicator of overall metallicity. After examining offsets in some relative abundances in DLAs, they determined corrections for the zero-depletion element abundances caused by nucleosynthesis effects in such systems, with some additional guidance from the trends seen in metal-poor stars in our Galaxy. After applying these corrections, they derived for each element the coefficients $A_{2}$ and $B_{2}$ that gave a best fit for the depletions that satisfied the equation $D=A_{2}+B_{2}[\mathrm{Zn} / \mathrm{Fe}]$.

In principle, we could compare our results to those of De Cia et al. (2016) using the parameter [Zn/Fe] as a common measure of depletion strengths. However, our determination for the trend of $[\mathrm{Zn} / \mathrm{Fe}]$ as a function of $F_{*}(\mathrm{SMC})$ has a large uncertainty - much larger than for our measurements of $D(\mathrm{Fe})$. Hence, as we did for the results of Tchernyshyov et al. (2015) in Section 9.2, we link our depletion sequence to that of De Cia et al. (2016) by using the depletions of Fe and find that their depletion parameter $[\mathrm{Zn} / \mathrm{Fe}]$ can be linked to ours using the expression $[\mathrm{Zn} / \mathrm{Fe}]=1.016 F_{*}(\mathrm{SMC})+0.744$. We then find that the transformations to our coefficients from theirs with subscripts $2, X$ for various elements $X$ (see their Table 3) are given by

$$
A_{X}=1.016 B_{2, X}
$$

and

$$
B_{X}=B_{2, X}\left(1.016 z_{X}+0.744\right)+A_{2, X} .
$$

As we did for the results of Tchernyshyov et al. (2015), we once again compare coefficients arising from the above two equations to those that we derived for the SMC, and we show the outcomes in Columns 4-7 of Table 7.

In agreement with the results from Ledoux et al. (2002), De Cia et al. (2016) find that Mn depletes less rapidly than $\mathrm{Fe}$, whereas we found that the difference between the two slopes was not significant. The pronounced trends for $[\mathrm{Mn} / \mathrm{Fe}]$ as a function of $[\mathrm{Zn} / \mathrm{Fe}]$ in DLAs may signify the existence of either some chemical evolution effect that has not yet been recognized or a depletion sequence that more closely matches the behavior in our Galaxy. The value for $A_{\mathrm{Mg}}$ in the DLAs seems to be midway between the Milky Way and SMC values for this slope parameter. The slope for the depletion trend of $\mathrm{Si}$ is only half as large that for $\mathrm{Fe}$, and the absolute value for $A_{\mathrm{P}}$ is quite small, in contrast to the findings for both the Milky Way and the SMC.

\subsection{A Possible Influence from the Abundance of Carbon}

In Section 5 we adopted the viewpoint that all of the elements that we considered had abundances that were uniformly below the solar abundances by about -0.65 dex, at least to within an envelope of apparent deviations of order 0.1 to 0.2 dex. It is natural to question why this dilute mixture of heavy elements (relative to $\mathrm{H}$ ) with an otherwise identical composition should deplete onto dust differently in the SMC than what we found in our Galaxy. The answer may lie in the abundance of the element carbon, which up to now we have not considered in this investigation. Carbon is a key constituent in polycyclic aromatic hydrocarbons (PAHs). Calculations by Weingartner \& Draine (1999) emphasize the probable importance for the binding of heavy elements onto very small PAHs. If the balance in the SMC between PAHs (or other carbonaceous compounds) and silicates differs from that of our Galaxy, then the mix of substrates upon which additional atoms can condense will be different. Differences in the chemical affinities of various elements on existing grains could influence the growth and destruction rates for the outer portions of the grains.

Determinations of stellar abundances in the SMC indicate that carbon may be more deficient than the other elements. Measurements of the features of C II and C III in two main-sequence B-type stars in the SMC and an application of a non-LTE analysis by Hunter et al. (2005) yielded $\log (\mathrm{C} / \mathrm{H})+12=7.42$, which is -1.01 dex below the solar abundance of carbon and -0.36 dex below our average of -0.65 dex that we adopted for all of the other elements. Other good determinations yielded even lower values for the SMC stellar abundances, e.g., 7.30 (Trundle et al. 2004 ; Dufton et al. 2005 ; Hunter et al. 2009) and 7.35 (Hunter et al. 2007). The outcomes for the $\mathrm{C}$ abundances are not surprising: stars that have $6.6 \lesssim \log (\mathrm{O} / \mathrm{H})+12 \lesssim 7.6$ (i.e., $-1.8 \lesssim[\mathrm{O} / \mathrm{H}] \lesssim-0.8)$ in the Milky Way halo likewise exhibit unusually low values of $[\mathrm{C} / \mathrm{O}]$, i.e., $[\mathrm{C} / \mathrm{O}] \approx-0.5$ (Akerman et al. 2004). Moreover, the deviation of $\mathrm{C}$ below the reduced 
abundances of other elements in the SMC seems to be duplicated in other dwarf galaxies with low metallicities. For instance, a study of nebular emission lines by Berg et al. (2016) found that when $\log (\mathrm{O} / \mathrm{H})+12 \leq 8.0,[\mathrm{C} / \mathrm{O}]$ reverts to a mean value of -0.36 , but with a large dispersion $(0.25 \mathrm{dex})$ from one system to the next. The more strongly reduced carbon abundances seen in the SMC, Milky Way halo stars, and other metal-poor systems may reflect the increasing importance of a secondary production of carbon in low and intermediate mass stars as the overall abundances approach the solar value.

Entirely different kinds of observations seem to support the aforementioned carbon abundance trend: the emission from the PAH features in the mid-infrared appear to undergo an abrupt weakening for galactic systems when the oxygen abundances appear to be diminished to values $\log (\mathrm{O} / \mathrm{H})+12 \lesssim 8.0$ (Wu et al. 2006 ; Draine et al. 2007 ; Engelbracht et al. 2008). In addition, if we accept the interpretation that the $2175 \AA$ extinction bump is likely to be caused by large carbon compounds (Draine 2003), the lack of this feature in the extinction curves associated with QSO absorption-line systems (Pei et al. 1991 ; York et al. 2006 ; Budzynski \& Hewett 2011 ; Khare et al. 2012) helps to reinforce our perception about the apparent extra deficiency of carbon in systems with low metallicities. However, studies of DLAs with extremely low metallicities $(\log (\mathrm{O} / \mathrm{H})+12 \lesssim 7)$ by Pettini et al. (2008), Penprase et al. (2010), and Cooke et al. (2011) revealed that when metal deficiencies become more extreme, the values of [C/O] begin to revert upward toward zero.

Much of what we said about distant, low-metallicity systems in general applies to the SMC. With one exception that we discussed in Section 7.4, more than half of the sight lines with $A_{V}>0.1$ through the SMC exhibit extinction curves that lack a detectable $2175 \AA$ bump (Hagen et al. 2016). Li \& Draine (2002) determined that PAH mid-IR emission from the interstellar medium of the SMC is relatively weak, which supports the interpretation that carbon compounds are deficient. Sandstrom et al. (2010) performed a more comprehensive investigation of the PAH emission using the IRAC, MIPS and IRS instruments on the Spitzer facility. They concluded that the fractional abundance of PAHs in the diffuse SMC material is low: the $q_{\mathrm{PAH}}$ index $^{8}$ averages about $0.6 \%$, and rises to $q_{\mathrm{PAH}} \sim 1-2 \%$ in the dense molecular regions. By comparison, $q_{\mathrm{PAH}} \approx 4.6 \%$ in the Milky Way (Draine \& Li 2007).

One challenge to our suggestion that a deficiency of carbon compounds may be responsible for the differences between the SMC and Milky Way depletions is our finding that the results for AzV 456 seem to fit well with the sequences for different elements that we found for other stars. However, as we pointed out in Section 7.4, the line of sight to this star shows an extinction curve that is unlike those of other SMC cases and closer to the behavior for Milky Way sight lines. From a different perspective, a map shown by Sandstrom et al. (2010) indicates that the stars AzV 18, AzV 26, and $\mathrm{AzV} 47$ are positioned in a region where $q_{P A H} \sim 1 \%$, which is higher than other regions where most of our stars are located. These stars do not seem to depart from the depletion sequences shown by the other ones. Nevertheless, one could argue that the gas cloud with enhanced values of $q_{\mathrm{PAH}}$ could be situated behind these three stars.

\section{SUMMARY}

Our principal objective was to investigate how rapidly different elements condense into the solid phase within the interstellar medium of the SMC. Since the chemical evolution of the SMC is probably similar to other galactic systems with moderately low metallicities at redshifts $z \lesssim 3.5$, our depletion sequences could serve as a guide for understanding the removal of elements from the gas phase. In turn, this information will aid in the determination of total element abundances in DLAs and sub-DLAs. In addition, the SMC depletions help us to understand better the compositions of dust grains in the SMC, which can be compared to those in our Galaxy.

The nature and results of our investigation are as follows:

1. We conducted observations of the spectra over the wavelength range $1123-2673 \AA$ for 14 hot stars in the SMC using the E140M and E230M echelle modes of the STIS instrument on the HST during the Cycle 22 observing session. We supplemented these data with existing UV spectra in the MAST archive for 3 other stars and column densities in the literature for one additional star. We derived column densities for Mg II, Si II, S II, Cr II, Mn II, Fe II, Ni II, and Zn II, and we made use of Ti II results published by Welty \& Crowther (2010). We obtained atomic and molecular hydrogen column densities toward our stars from Welty et al. (2012).

2. For the total element abundance standards against which to compare the interstellar abundances, we have examined the findings reported in the literature for the abundances in young stars within the SMC. For investigations that we deemed to be most reliable, we found that the dispersions in outcomes for most elements were on the

\footnotetext{
8 This index for the concentration of PAHs is defined by Draine \& Li (2007) and Draine et al. (2007) as the mass fraction of dust that consists of PAH particles with less than $10^{3}$ carbon atoms.
} 
order of $0.15 \mathrm{dex}$, although we recognize that additional errors of an unknown systematic nature could exist for certain elements. Except for carbon, the abundances of $\alpha$-process with respect to Fe-group elements seemed not to differ appreciably from the solar abundance ratios. We found no compelling reason for adopting an SMC abundance pattern that was more complex than a uniform deficiency of -0.65 dex below the solar abundances defined by Asplund et al. (2009).

3. We presented in Table 3 column densities and the depletion outcomes defined in Eq. 1 for all measurements where the absorption features were strong enough to measure above the noise but not so strong that they were too saturated to yield useful results. These restrictions precluded our being able to measure the abundances of $\mathrm{B}, \mathrm{C}, \mathrm{N}, \mathrm{O}, \mathrm{P}, \mathrm{Cu}, \mathrm{Ge}, \mathrm{Ga}$, and $\mathrm{Kr}$, even though they had transitions within our wavelength coverage.

4. We found that most of the possible pairs of elements exhibited tight linear relationships with each other in their depletions. In Section 7.1 we analyzed the strengths of the correlations and found that many of them (23 out of 36) were significant at or above the $95 \%$ confidence level. The remaining cases showing weaker correlations could mostly be attributed to larger uncertainties in the measurements, reduced dynamic ranges, or small sample sizes. We found that the loose correlation between the depletions of $\mathrm{Zn}$ and Fe appear to differ from the much tighter correlation for those of $\mathrm{Ti}$ and $\mathrm{Fe}$ at the $95 \%$ confidence level, which suggests that there may be some variation in the $\mathrm{Zn}$ and $\mathrm{Ti}$ concentrations in dust in different regions. Another possibility is that possibly different nucleosynthesis sources, combined with poor mixing in the SMC ISM, may explain the apparent variation in $\mathrm{Zn}$ depletions.

5. Following a scheme introduced by Jenkins (2009) for depletions in our Galaxy, we developed a unified description of depletions in terms of a generalized sight-line depletion parameter $F_{*}(\mathrm{SMC})$ and two coefficients for each element that described a best-fit slope and offset in the linear relationship with this parameter (see Eq. 3). We used Fe depletions to link the scale for $F_{*}(\mathrm{SMC})$ to that of $F_{*}(\mathrm{MW})$ in the Milky Way. Values of $F_{*}(\mathrm{SMC})$ for each star in our program are listed in Table 5, and the coefficients $A_{X}, B_{X}$ and $z_{X}$ for each element $X$ are listed in the right-hand portion of Table 6. From the $\chi^{2}$ values associated with the fits, we found that the elements $\mathrm{Mg}$, $\mathrm{Si}$, and $\mathrm{Zn}$ showed exceptionally low values for the probabilities of obtaining worse fits to the $F_{*}(\mathrm{SMC})$ trends, indicating that either our measurement uncertainty estimates were too low or that there are true variations in relative abundances beyond those attributable to our regime of generalized depletions.

6. We identified two stars that were conspicuously different from the others in our survey. The star AzV 456 has an unusually high abundance of molecules in front of it and exhibits a UV extinction curve that is more similar to that of the Milky Way than the one typically found for matter in the SMC. The depletions toward this star are strong, but they seem to lie on extrapolations of the trends seen for other stars. The star Sk 191 shows even more depletion than AzV 456. This star is located midway between the wing of the SMC and the Magellanic Bridge. While one might be suspicious that the overall abundances of material near this star may be lower than usual for the SMC, the star itself shows element abundances close to others in the SMC. Moreover, there is an indication from a marginal detection of the semi-forbidden O I absorption feature at $1356 \AA$ that the total ISM abundances are not depressed below those seen elsewhere in the SMC.

7. We examined differential changes in dust elemental compositions as a function of $F_{*}(\mathrm{SMC})$ and found that, aside from the effect of a lower overall metal abundance, the general pattern of consumption of the different atoms was about the same as in the Milky Way, except for the few elements $\mathrm{Mg}, \mathrm{Ti}$, and $\mathrm{Mn}$, where their respective values of $A_{X}$ for the SMC differed from their counterparts in the Milky Way. One important feature of this differential measurement is that the outcome is not dependent on knowing the stellar reference abundance.

8. Our study overlaps the findings of Tchernyshyov et al. (2015) for the elements $\mathrm{Si}, \mathrm{Cr}, \mathrm{Fe}$, and $\mathrm{Zn}$. We converted their coefficients to our system and found that, with the exceptions of $B_{\mathrm{Si}}$ and $A_{\mathrm{Zn}}$, the values overlap to within the uncertainties of both determinations. We did not include phosphorous in our survey, so we converted the values of $A_{\mathrm{P}}$ and $\delta(\mathrm{P})_{0}$ of Tchernyshyov et al. (2015) to our $A_{\mathrm{P}}$ and $B_{\mathrm{P}}$ for an assumed value $z_{\mathrm{P}}=0.15$ (see Table 7).

9. The relative amount of dust in the form of PAHs in the SMC is significantly lower than in the Milky Way. If the chemical affinities of some of our elements on PAHs differ from those with other types of dust, such as silicates, we might have an explanation for why some of our depletion trends are either steeper or shallower than 
in the Milky Way. Other possible influences might include a generally more intense UV radiation field, lower $\mathrm{H}_{2}$ fractions, or greater $\mathrm{H}_{2}$ rotational excitations (Tumlinson et al. 2002). Otherwise, it is difficult to understand why the dilute mix (relative to hydrogen) of elements would behave differently than what we observe locally.

Support for the HST observing program nr. 13778 was provided by NASA through a grant from the Space Telescope Science Institute (STScI), which is operated by the Associations of Universities for Research in Astronomy, Incorporated, under NASA contract NAS5-26555. Some of the data presented in this paper were obtained from the Mikulski Archive for Space Telescopes (MAST) maintained by the STScI. G.W. thanks the University of Washington for providing office space and a desktop computer to emeritus professors. The authors thank D. E. Welty and B. T. Draine for useful discussions. Dr. Welty also furnished the error estimates for the hydrogen column densities, and S. Woosley gave us advice on the nucleosynthesis of $\mathrm{Zn}$. We thank the anonymous referee for undergoing a careful reading of our paper and providing thoughtful comments.

\section{Facility: HST (STIS), HST (GHRS)}

\section{Software: FITEXY (Press et al. 2007)}

\section{REFERENCES}

Akerman, C. J., Carigi, L., Nissen, P. E., Pettini, M., \& Asplund, M. 2004, A\&A, 414, 931

Asplund, M., Grevesse, N., Sauval, A. J., \& Scott, P. 2009, ARA\&A, 47, 481

Azzopardi, M., \& Vigneau, J. 1982, A\&AS, 50, 291

Berg, D. A., Skillman, E. D., Henry, R. B. C., Erb, D. K., \& Carigi, L. 2016, ApJ, 827, 126

Blanco-Cuaresma, S., Nordlander, T., Heiter, U., et al. 2016, arXiv: 1609.09071

Bohlin, R. C. 1975, ApJ, 200, 402

Boulanger, F., Prevot, M. L., \& Gry, C. 1994, A\&A, 284, 956

Bromage, G. E., \& Nandy, K. 1983, MNRAS, 204, 29P

Budzynski, J. M., \& Hewett, P. C. 2011, MNRAS, 416, 1871

Burbidge, E. M., Burbidge, G. R., Fowler, W. A., \& Hoyle, F. 1957, RvMP, 29, 547

Calura, F., Matteucci, F., \& Vladilo, G. 2003, MNRAS, 340, 59

Cameron, A. G. W. 1957, PASP, 69, 201

Cartledge, S. I. B., Clayton, G. C., Gordon, K. D., et al. 2005, ApJ, 630, 355

Cassinelli, J. P., Cohen, D. H., MacFarlane, J. J., et al. 1996, ApJ, 460,949

Chen, H.-W., Kennicutt, R. C., Jr., \& Rauch, M. 2005, ApJ, 620, 703

Chiappini, C., Matteucci, F., Beers, T. C., \& Nomoto, K. 1999, ApJ, 515, 226

Christensen, L., Schulte-Ladbeck, R. E., Sánchez, S. F., et al. 2005 , A\&A, 429, 477

Cooke, R., Pettini, M., Steidel, C. C., Rudie, G. C., \& Nissen, P. E. 2011, MNRAS, 417, 1534

Cox, N. L. J., Cordiner, M. A., Ehrenfreund, P., et al. 2007, A\&A, 470,941

De Cia, A., Ledoux, C., Mattsson, L., et al. 2016, A\&A, 596, A97

Den Hartog, E. A., Lawler, J. E., Sobeck, J. S., Sneden, C., \& Cowan, J. J. 2011, ApJS, 194, 35

Dessauges-Zavadsky, M., Calura, F., Prochaska, J. X., D'Odorico, S., \& Matteucci, F. 2004, A\&A, 416, 79

Dessauges-Zavadsky, M., Prochaska, J. X., D'Odorico, S., Calura, F., \& Matteucci, F. 2006, A\&A, 445, 93

Dessauges-Zavadsky, M., Calura, F., Prochaska, J. X., D’Odorico, S., \& Matteucci, F. 2007, A\&A, 470, 431

Draine, B. T., \& Lee, H. M. 1984, ApJ, 285, 89

Draine, B. T. 2003, ARA\&A, 41, 241

Draine, B. T., \& Li, A. 2007, ApJ, 657, 810

Draine, B. T., Dale, D. A., Bendo, G., et al. 2007, ApJ, 663, 866

Dufton, P. L., Ryans, R. S. I., Trundle, C., et al. 2005, A\&A, 434, 1125

Dufton, P. L., Ryans, R. S. I., Thompson, H. M. A., \& Street, R. A. 2008, MNRAS, 385, 2261

Dwek, E. 2016, ApJ, 825, 136

Ellison, S. L., Kewley, L. J., \& Mallén-Ornelas, G. 2005, MNRAS, 357,354
Engelbracht, C. W., Rieke, G. H., Gordon, K. D., et al. 2008, ApJ 678,804

Evans, I. N., \& Dopita, M. A. 1985, ApJS, 58, 125

Evans, I. N. 1991, ApJS, 76, 985

Fox, A., Richter, P., \& Fechner, C. 2014, A\&A, 572, A102

Fox, A. J., Savage, B. D., \& Wakker, B. P. 2005, AJ, 130, 2418

Gardiner, L. T., \& Noguchi, M. 1996, MNRAS, 278, 191

Gordon, K. D., \& Clayton, G. C. 1998, ApJ, 500, 816

Gordon, K. D., Clayton, G. C., Misselt, K. A., Landolt, A. U., \& Wolff, M. J. 2003, ApJ, 594, 279

Guber, C. R., \& Richter, P. 2016, A\&A, 591, A137

Habing, H. 1969, BAN, 20, 177

Hagen, L. M. Z., Siegel, M. H., Hoversten, E. A., et al. 2016, arXiv: 1611.00064

Hammer, F., Yang, Y. B., Flores, H., Puech, M., \& Fouquet, S. 2015, ApJ, 813, 110

Hinkel, N. R., Young, P. A., Pagano, M. D., et al. 2016, arXiv: 1607.03130

Hou, J. L., Boissier, S., \& Prantzos, N. 2001, A\&A, 370, 23

Hunter, I., Dufton, P. L., Ryans, R. S. I., et al. 2005, A\&A, 436, 687

Hunter, I., Dufton, P. L., Smartt, S. J., et al. 2007, A\&A, 466, 277

Hunter, I., Brott, I., Langer, N., et al. 2009, A\&A, 496, 841

Hutchings, J. B. 1982, ApJ, 255, 70

Izotov, Y. I., \& Thuan, T. X. 1999, ApJ, 511, 639

Jenkins, E. 2013, in The Life Cycle of Dust in the Universe: Observations, Theory, and Laboratory Experiments, eds. A. Anderssen, et al. (Taipei, Taiwan: Proceedings of Science), 15

Jenkins, E. B., Savage, B. D., \& Spitzer, L. 1986, ApJ, 301, 355

Jenkins, E. B. 1996, ApJ, 471, 292

Jenkins, E. B., \& Tripp, T. M. 2006, ApJ, 637, 548

Jenkins, E. B. 2009, ApJ, 700, 1299

Kalberla, P. M. W., McClure-Griffiths, N. M., Pisano, D. J., et al. 2010, A\&A, 521, A17

Khare, P., Vanden Berk, D., York, D. G., Lundgren, B., \& Kulkarni, V. P. 2012, MNRAS, 419, 1028

Kisielius, R., Kulkarni, V. P., Ferland, G. J., Bogdanovich, P., \& Lykins, M. L. 2014, ApJ, 780, 76

Kisielius, R., Kulkarni, V. P., Ferland, G. J., et al. 2015, ApJ, 804, 76

Kobayashi, C., Umeda, H., Nomoto, K. i., Tominaga, N., \& Ohkubo, T. 2006, ApJ, 653, 1145

Koenigsberger, G., Georgiev, L., Peimbert, M., et al. 2001, AJ, 121,267

Korn, A. J., Becker, S. R., Gummersbach, C. A., \& Wolf, B. 2000, A\&A, 353, 655

Kulkarni, V. P., Fall, S. M., \& Truran, J. W. 1997, ApJ, 484, L7

Kulkarni, V. P., Som, D., Morrison, S., et al. 2015, ApJ, 815, 24

Ledoux, C., Bergeron, J., \& Petitjean, P. 2002, A\&A, 385, 802

Lee, J. K., Rolleston, W. R. J., Dufton, P. L., \& Ryans, R. S. I. 2005, A\&A, 429, 1025 
Lehner, N., Sembach, K. R., Dufton, P. L., Rolleston, W. R. J., \& Keenan, F. P. 2001, ApJ, 551, 781

Lehner, N., Howk, J. C., Keenan, F. P., \& Smoker, J. V. 2008, ApJ, 678, 219

Levshakov, S. A., Agafonova, I. I., Molaro, P., Reimers, D., \& Hou, J. L. 2009, A\&A, 507, 209

Li, A., \& Draine, B. T. 2002, ApJ, 576, 762

Lodders, K. 2003, ApJ, 591, 1220

Lopez, S., Reimers, D., Gregg, M. D., et al. 2005, ApJ, 626, 767

Lu, L., Sargent, W. L. W., Barlow, T. A., Churchill, C. W., \& Vogt, S. S. 1996, ApJS, 107, 475

Luck, R. E., \& Lambert, D. L. 1992, ApJS, 79, 303

Luck, R. E., Moffett, T. J., Barnes, T. G., III, \& Gieren, W. P. 1998, AJ, 115, 605

Maíz Apellániz, J., \& Rubio, M. 2012, A\&A, 541, A54

Mallouris, C., Welty, D. E., York, D. G., et al. 2001, ApJ, 558, 133

Mallouris, C. 2003, ApJS, 147, 265

Matteucci, F. 2003, The Chemical Evolution of the Galaxy, Vol. 253 (Dordrecht: Springer)

McWilliam, A. 1997, ARA\&A, 35, 503

Meiring, J. D., Lauroesch, J. T., Kulkarni, V. P., et al. 2009, MNRAS, 397, 2037

Morrison, S., Kulkarni, V. P., Som, D., et al. 2016, ApJ, 830, 158

Morton, D. C. 1975, ApJ, 197, 85

- 2003, ApJS, 149, 205

Mucciarelli, A. 2014, AN, 335, 79

Murphy, M. T., \& Bernet, M. L. 2016, MNRAS, 455, 1043

Pei, Y. C., Fall, S. M., \& Bechtold, J. 1991, ApJ, 378, 6

Penprase, B. E., Prochaska, J. X., Sargent, W. L. W., Martinez, I. T., \& Beeler, D. J. 2010, ApJ, 721, 1

Péroux, C., Bouché, N., Kulkarni, V. P., York, D. G., \& Vladilo, G. 2011, MNRAS, 410, 2237

Péroux, C., Bouché, N., Kulkarni, V. P., York, D. G., \& Vladilo, G. 2012, MNRAS, 419, 3060

Péroux, C., Kulkarni, V. P., \& York, D. G. 2014, MNRAS, 437, 3144

Pettini, M., Smith, L. J., Hunstead, R. W., \& King, D. L. 1994, ApJ, 426, 79

Pettini, M., Smith, L. J., King, D. L., \& Hunstead, R. W. 1997, ApJ, 486, 665

Pettini, M., Ellison, S. L., Steidel, C. C., \& Bowen, D. V. 1999, ApJ, 510, 576

Pettini, M., Ellison, S. L., Steidel, C. C., Shapley, A. E., \& Bowen, D. V. 2000, ApJ, 532, 65

Pettini, M., Zych, B. J., Steidel, C. C., \& Chaffee, F. H. 2008, MNRAS, 385, 2011

Press, W. H., Teukolsky, S. A., Vetterling, W. T., \& Flannery, B. P. 2007, Numerical Recipes, The Art of Scientific Computing (3rd ed.; Cambridge: Cambridge Univ. Press)

Prevot, M. L., Lequeux, J., Prevot, L., Maurice, E., \& RoccaVolmerange, B. 1984, A\&A, 132, 389

Prochaska, J. X., \& Wolfe, A. M. 1999, ApJS, 121, 369

-. 2002, ApJ, 566, 68

Prochaska, J. X., Howk, J. C., \& Wolfe, A. M. 2003, Natur, 423, 57

Prochaska, J. X., O’Meara, J. M., Fumagalli, M., Bernstein, R. A., \& Burles, S. M. 2015, ApJS, 221, 2

Quiret, S., Péroux, C., Zafar, T., et al. 2016, MNRAS, 458, 4074

Rafelski, M., Wolfe, A. M., Prochaska, J. X., Neeleman, M., \& Mendez, A. J. 2012, ApJ, 755, 89

Rafelski, M., Neeleman, M., Fumagalli, M., Wolfe, A. M., \& Prochaska, J. X. 2014, ApJ, 782, L29

Rodríguez, E., Petitjean, P., Aracil, B., Ledoux, C., \& Srianand, R. 2006, A\&A, 446, 791

Rolleston, W. R. J., Dufton, P. L., McErlean, N. D., \& Venn, K. A. 1999, A\&A, 348, 728

Rolleston, W. R. J., Venn, K., Tolstoy, E., \& Dufton, P. L. 2003, A\&A, 400, 21
Roth, K. C., \& Blades, J. C. 1997, ApJ, 474, L95

Russell, S. C., \& Dopita, M. A. 1992, ApJ, 384, 508

Sandstrom, K. M., Bolatto, A. D., Draine, B. T., Bot, C., \& Stanimirović, S. 2010, ApJ, 715, 701

Sarazin, C. L. 1977, ApJ, 211, 772

Savage, B. D., \& Sembach, K. R. 1991, ApJ, 379, 245

-. 1996, ARA\&A, 34, 279

Savaglio, S. 2001, in The Extragalactic Infrared Background and its Cosmological Implications, eds. M. Harwit, \& M. G. Hauser (IAU Symp. 204), 307- 321

Schulte-Ladbeck, R. E., König, B., Miller, C. J., et al. 2005, ApJ, 625, L79

Sembach, K. R., \& Savage, B. D. 1992, ApJS, 83, 147

Sofia, U. J., Gordon, K. D., Clayton, G. C., et al. 2006, ApJ, 636, 753

Som, D., Kulkarni, V. P., Meiring, J., et al. 2013, MNRAS, 435, 1469

Som, D., Kulkarni, V. P., Meiring, J., et al. 2015, ApJ, 806, 25

Suess, H. E., \& Urey, H. C. 1956, RvMP, 28, 53

Sukhbold, T., Ertl, T., Woosley, S. E., Brown, J. M., \& Janka, H. T. 2016, ApJ, 821, 38

Tchernyshyov, K., Meixner, M., Seale, J., et al. 2015, ApJ, 811, 78

Thuan, T. X., Izotov, Y. I., \& Lipovetsky, V. A. 1995, ApJ, 445, 108

Timmes, F. X., Woosley, S. E., \& Weaver, T. A. 1995, ApJS, 98, 617

Tolstoy, E., Hill, V., \& Tosi, M. 2009, ARA\&A, 47, 371

Trundle, C., Lennon, D. J., Puls, J., \& Dufton, P. L. 2004, A\&A, 417,217

Trundle, C., Dufton, P. L., Hunter, I., et al. 2007, A\&A, 471, 625

Tumlinson, J., Shull, J. M., Rachford, B. L., et al. 2002, ApJ, 566, 857

Vallerga, J. V., \& Welsh, B. Y. 1995, ApJ, 444, 702

Venn, K. A. 1999, ApJ, 518, 405

Vladilo, G., Centurión, M., Bonifacio, P., \& Howk, J. C. 2001, ApJ, 557, 1007

Vladilo, G. 2002, ApJ, 569, 295

Vladilo, G. 2004, A\&A, 421, 479

Wallerstein, G., \& Goldsmith, D. 1974, ApJ, 187, 237

Weingartner, J. C., \& Draine, B. T. 1999, ApJ, 517, 292

Weingartner, J. C., \& Draine, B. T. 2001, ApJ, 548, 296

Welty, D. E., Lauroesch, J. T., Blades, J. C., Hobbs, L. M., \& York, D. G. 1997, ApJ, 489, 672

—. 2001, ApJ, 554, L75

Welty, D. E., Federman, S. R., Gredel, R., Thorburn, J. A., \& Lambert, D. L. 2006, ApJS, 165, 138

Welty, D. E., \& Crowther, P. A. 2010, MNRAS, 404, 1321

Welty, D. E., Xue, R., \& Wong, T. 2012, ApJ, 745, 173

Welty, D. E., Howk, J. C., Lehner, N., \& Black, J. H. 2013, MNRAS, 428, 1107

Welty, D. E., Lauroesch, J. T., Wong, T., \& York, D. G. 2016, ApJ, 821, 118

Wheeler, J. C., Sneden, C., \& Truran, J. W., Jr. 1989, ARA\&A, 27,279

Wiseman, P., Schady, P., Bolmer, J., et al. 2016, arXiv: 1607.00288

Wolfe, A. M., Gawiser, E., \& Prochaska, J. X. 2005, ARA\&A, 43, 861

Woosley, S. E., Heger, A., \& Weaver, T. 2002, RvMP, 74, 1015

Wu, Y., Charmandaris, V., Hao, L., et al. 2006, ApJ, 639, 157

York, D. G., Khare, P., Vanden Berk, D., et al. 2006, MNRAS, 367,945

Zonca, A., Casu, S., Mulas, G., Aresu, G., \& Cecchi-Pestellini, C. 2015, ApJ, 810, 70

Zubko, V., Dwek, E., \& Arendt, R. G. 2004, ApJS, 152, 211 